\title{
Central limit theorem for a class of globally correlated random variables
}

\author{
Adrián A. Budini \\ Consejo Nacional de Investigaciones Científicas y Técnicas (CONICET), \\ Centro Atómico Bariloche, Avenida E. Bustillo Km 9.5, (8400) Bariloche, \\ Argentina, and Universidad Tecnológica Nacional (UTN-FRBA), \\ Fanny Newbery 111, (8400) Bariloche, Argentina
}

(Dated: August 26, 2021)

\begin{abstract}
The standard central limit theorem with a Gaussian attractor for the sum of independent random variables may lose its validity in presence of strong correlations between the added random contributions. Here, we study this problem for similar interchangeable globally correlated random variables. Under these conditions, a hierarchical set of equations is derived for the conditional transition probabilities. This result allows us to define different classes of memory mechanisms that depend on a symmetric way on all involved variables. Depending on the correlation mechanisms and single statistics, the corresponding sums are characterized by distinct statistical probability densities. For a class of urn models it is also possible to characterize their domain of attraction which, as in the standard case, is parametrized by the probability density of each random variable. Symmetric and asymmetric $q$-Gaussian attractors $(q<1)$ are a particular case of these models.
\end{abstract}

PACS numbers: 02.50.-r, 05.40.-a, 89.75.Da

\section{INTRODUCTION}

The standard central limit theorem (CLT) is a cornerstone of probability theory [1-4]. It establishes that a sum of independent (identical) random variables, under a suitable rescaling, converges to a Gaussian distribution. It plays a fundamental role in the formulation of statistical thermodynamics and also provide a rigorous basis for assuming Gaussian statistics for describing fluctuations in equilibrium and nonequilibrium systems.

There exist a few remarkable examples where the standard CLT was generalized. The Gaussian attractor arises when considering independent random variables with a finite second moment. As is well known, when this condition is raised up the attractor becomes a Levy distribution [5]. On the other hand, Gumbel distribution arises from the study of extreme value statistics and describes the fluctuations of the largest value in a large set of identically distributed independent random variables $[6]$. Interestingly, this problem can in general be related with the statistics of random sums of correlated random variables 7]. Departure from Gaussian statistics was also analyzed for global correlations where the characteristic function of the total sum is defined by a non-multiplicative Fourier structure [8].

Recently it was argued that the presence of global correlations in stationary equilibrium and nonequilibrium systems is a situation where nonextensive statistical mechanics may applies [9 12]. Consistently, many theoretical effort was devoted to finding global memory mechanisms that lead to attractors defined by $q$-Gaussian probability densities [13 18]. These statistical objects also arise from maximizing Tsallis entropy [9], from superstatistical models [19], as well as from specific transformations of Gamma distributed random variables [20].

Global correlations are a mechanism that may lead to departures from Gaussian statistics. Nevertheless, estab- lishing a generalization of the CLT on the basis of only this feature is a formidable task. In fact, to our knowledge, there not exist general rigorous mathematical criteria for splitting correlations in weak ones (leading to Gaussian statistics) and stronger ones (departure from normal distribution). Therefore, as in the previous literature [7-18], one is naturally forced to study particular cases. Of special interest is to find generalizations that rely on simple correlation mechanisms or symmetries, which in turn also allow defining or studying its domain of attraction. In general, this last issue is hard to solve.

In this paper we analyze the departure from the standard CLT for a specific class of global correlations. Similar interchangeable random variables [21 25] are considered. This property or symmetry, originally introduced by de Finetti 21] in probability theory, is defined by random variables whose joint probability density is invariant under arbitrary permutations of its arguments.

The main goal is twofold. First, we give a general characterization of possible correlations mechanisms consistent with interchangeability. This objective is achieved by characterizing the correlations not through the joint probability densities but through the transition probabilities. These functions say us how the probability density of a given variable depends on the previous values assumed by the rest of the random variables. We demonstrate that these objects obey a set of hierarchical equations that resemble a Chapman-Kolmogorov equation for Markovian chains 2-4]. From this result we construct different correlation models which allow us to achieve the second main goal, that is, the characterization of the departure from Gaussian statistics as well as to study their domain of attraction. For a class of urn models [26 29], we demonstrate that their domain of attraction is as wide as in the standard case. Asymmetric and symmetric $q$ Gaussian attractors [20] with $q<1$ arise as a particular 
case of these urn models.

The paper is outlined as follows. In Sec. II, based on the interchangeability property of the joint probabilities, we derive a hierarchical set of equations for the transition probabilities. Sec. III is devoted to finding different solutions to the previous equations, which are based on a generalization of Pólya urn scheme [26 29]. In Sec. IV, departure from Gaussian statistics and their basin of attraction are analyzed. In Sec. V we provide the Conclusions. In the Appendixes we show some calculus details and study other correlation models (additive memory, de Finetti representation, Blackwell-MacQueen urn scheme).

\section{HIERARCHY OF TRANSITION PROBABILITIES FOR SIMILAR INTERCHANGEABLE RANDOM VARIABLES}

A set of $n$ random variables $X_{1}, X_{2}, \cdots X_{n}$, can be characterized by the $n$-joint probability distribution $P_{n}\left(x_{1}, x_{2}, \cdots x_{n}\right)$, which defines the probability that each variable falls in an infinitesimal range $d x_{i}$ around $x_{i}$.

Similar interchangeable variables are defined by the following two symmetries. Similarity (or scale invariance [9]) means that for any $n$ it is fulfilled the relation

$$
P_{n-1}\left(x_{1}, x_{2}, \cdots x_{n-1}\right)=\int d x_{n} P_{n}\left(x_{1}, x_{2}, \cdots x_{n}\right) .
$$

Therefore, the joint probability density of the subset of $(n-1)$ random variables coincides with the marginal distribution corresponding to $n$ variables. On the other hand, interchangeability is defined by the invariance of the joint probability density under arbitrary permutations of its arguments,

$$
P_{n}\left(\cdots, x_{k}, \cdots, x_{l}, \cdots\right)=P_{n}\left(\cdots, x_{l}, \cdots, x_{k}, \cdots\right),
$$

that is, for any $k$ and $l$ in the interval $(1,2, \cdots n)$, the joint probability density does not change under the (arbitrary) interchange $x_{k} \leftrightarrow x_{l}$. These relations are assumed valid for all values of $n$. Notice that in particular the previous two conditions imply that all random variables $\left\{X_{i}\right\}_{i=1}^{n}$ are characterized by the same distribution, $P_{1}(x)$.

The joint probability density $P_{n}\left(x_{1}, x_{2}, \cdots x_{n}\right)$ completely characterizes the random variables $\left\{X_{i}\right\}_{i=1}^{n}$. Nevertheless, an extra aspect is lighted by introducing a conditional probability density defined by the relation

$P_{n}\left(x_{1}, \cdots x_{n}\right)=P_{n-1}\left(x_{1}, \cdots x_{n-1}\right) T_{n-1}\left(x_{1}, \cdots x_{n-1} \mid x_{n}\right)$.

Hence, the function $T_{n-1}\left(x_{1}, \cdots x_{n-1} \mid x_{n}\right)$ gives the probability density of the variable $X_{n}$ given that the previous ones $\left\{X_{i}\right\}_{i=1}^{n-1}$ assumed the values $x_{1}, \cdots x_{n-1}$. By definition, it satisfies the normalization condition $\int d x_{n} T_{n-1}\left(x_{1}, \cdots x_{n-1} \mid x_{n}\right)=1$.

From Eq. (3), iteratively it follows

$$
\begin{aligned}
P_{n}\left(x_{1}, \cdots x_{n}\right)= & P_{1}\left(x_{1}\right) T_{1}\left(x_{1} \mid x_{2}\right) T_{2}\left(x_{1}, x_{2} \mid x_{3}\right) \cdots \\
& \cdots \times T_{n-1}\left(x_{1}, \cdots x_{n-1} \mid x_{n}\right) .
\end{aligned}
$$

Therefore, the set of functions $T_{k}\left(x_{1}, \cdots x_{k} \mid x_{k+1}\right)$, with $k=1, \cdots n-1$ provide the same information than the $n$-joint probability density. Furthermore, from Eq. (4) one can easily read how the correlations between the random variables are build up. In fact, having an explicit expression for the transition probabilities it is possible to numerically generate the corresponding realizations of the correlated variables $\left\{X_{i}\right\}_{i=1}^{n}$.

The main problem that we solve in this section is to determine which set of transition probabilities are consistent with the similarity and interchangeability properties. Given an arbitrary distribution $P_{1}\left(x_{1}\right)$, the symmetry does not impose any condition. For $n=2$, interchangeability implies $P_{2}\left(x_{1}, x_{2}\right)=P_{2}\left(x_{2}, x_{1}\right)$, or equivalently $P_{1}\left(x_{1}\right) T_{1}\left(x_{1} \mid x_{2}\right)=P_{1}\left(x_{2}\right) T_{1}\left(x_{2} \mid x_{1}\right)$. After integration, and by using the similarity property, it follows the condition

$$
\int d x_{1} P_{1}\left(x_{1}\right) T_{1}\left(x_{1} \mid x_{2}\right)=P_{1}\left(x_{2}\right) .
$$

By using a similar procedure, $T_{2}\left(x_{1}, x_{2} \mid x_{3}\right)$ must to fulfill

$$
T_{1}\left(x_{1} \mid x_{3}\right)=\int d x_{2} T_{1}\left(x_{1} \mid x_{2}\right) T_{2}\left(x_{1}, x_{2} \mid x_{3}\right) .
$$

For higher conditional probabilities densities (see Appendix $[$ A), the following relations

$$
\begin{aligned}
T_{n-1}\left(x_{1} \cdots x_{n-1} \mid x_{n+1}\right)= & \int d x_{n} T_{n-1}\left(x_{1}, \cdots x_{n-1} \mid x_{n}\right) \\
& \times T_{n}\left(x_{1}, \cdots x_{n} \mid x_{n+1}\right), \quad(7)
\end{aligned}
$$

must to be fulfilled for all values of $n$. Furthermore, the function $T_{n}\left(x_{1} \cdots x_{n} \mid x_{n+1}\right)$ must to be symmetric in the conditional arguments $x_{1} \cdots x_{n}$, that is, it is invariant under arbitrary permutations of its arguments. The hierarchical set of equations defined by (7) is the main result presented in this section.

If $T_{n-1}\left(x_{1}, \cdots x_{n-1} \mid x_{n+1}\right)$, for all values of $n$, does not depends on the previous values $x_{1}, \cdots x_{n-1}$, it follows $T_{n}\left(x_{1}, \cdots x_{n} \mid x_{n+1}\right)=P_{1}\left(x_{n+1}\right)$, that is, we recover the case of independent identical random variables. Notice that interchangeability implies that $T_{n-1}\left(x_{1} \cdots x_{n-1} \mid x_{n}\right)$ depends symmetrically on the previous arguments $x_{1} \cdots x_{n-1}$. Therefore, transition probabilities that only depend on one previous value, with a dependence that is independent on the number of previous events, $T_{n-1}\left(x_{1} \cdots x_{n-1} \mid x_{n}\right)=T_{n-1}\left(x_{n-1} \mid x_{n}\right)=$ $T\left(x_{n-1} \mid x_{n}\right)$, are not consistent with interchangeability. This case corresponds to stationary Markov chains. In fact, the unique transition probability $T(x \mid y)$ should to satisfy [Eq. (5)]

$$
\int d x P_{1}(x) T(x \mid y)=P_{1}(y),
$$

while by imposing the previous conditions on Eq. (17), it follows

$$
T(x \mid y)=\int d x^{\prime} T\left(x \mid x^{\prime}\right) T\left(x^{\prime} \mid y\right)
$$


The stationary property is given by Eq. (8), while the Markov property is defined by the Chapman-Kolmogorov relation Eq. (9). In fact, the next value (future) depends on the previous value (present state), but not on the manner in which the present state has emerged from previous ones (the past).

In the following section we search solutions of Eq. (7) where the transition probabilities are based on a Pólya urn scheme. In the Appendixes we studied other solutions that also depend in the same manner on all previous values taken by the random variables, that is, global correlations. For example an additive memory assumption $T_{n}\left(x_{1} \cdots x_{n} \mid x_{n+1}\right)=\mathcal{T}_{n}\left(x_{1}+x_{2} \cdots+x_{n} \mid x_{n+1}\right)$ (Appendix B) leads to consistent solutions for Gaussian and classical spin variables. A generalized de Finetti representation is analyzed in Appendix C

\section{URN SCHEMES}

Urn models are examples of random variables defined through their transition probabilities [2, 26]. Pólya urn scheme generate interchangeable random variables 2729]. Below we review this scheme, which gives us the basis for constructing a generalization consistent with interchangeability.

\section{A. Pólya Urn scheme}

The standard Pólya urn scheme can be seen as a particular case of the Blackwell-MacQueen urn scheme [27, 28], which in turn is related to the "Chinese restaurant process" [28, 29]. In the present context, it is defined by an arbitrary distribution $P_{1}(x)$, while the transition probabilities are

$$
T_{n}\left(x_{1}, \cdots x_{n} \mid x\right)=\frac{\lambda P_{1}(x)+\sum_{i=1}^{n} \delta\left(x-x_{i}\right)}{n+\lambda} .
$$

Here, $\lambda$ is dimensionless positive parameter, while $\delta(x)$ is the delta Dirac function. When $\lambda \rightarrow \infty$, identical independent random variables are recovered, while the limit $\lambda \rightarrow 0$ leads to a fully correlated case, that is, after the first random value the next ones assume the same value.

After a simple algebra it is possible to proof that the set of functions defined by Eq. (10) satisfy Eq. (5), as well as the hierarchical set of conditions corresponding to interchangeability, Eq. (7). In Appendix D, we analyze the departure from the standard CLT for this model.

The Pólya urn scheme corresponds to the particular case in which the random variables $\left\{X_{i}\right\}$ are discrete. Hence, we write

$$
P_{1}(x)=\sum_{\mu=1}^{M} q_{\mu} \delta\left(x-x_{\mu}\right),
$$

where $\left\{x_{\mu}\right\}_{\mu=1}^{M}$ is the set of $M$ possible values and $\left\{q_{\mu}\right\}_{\mu=1}^{M}$ are the corresponding weights (probabilities),

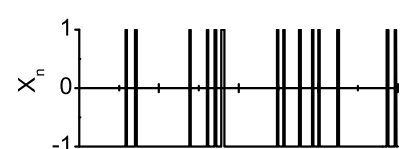

(a)
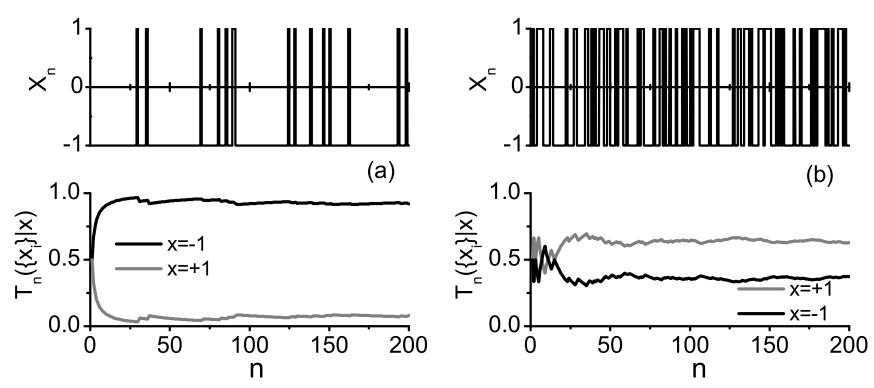

(b)
FIG. 1: Two realizations [(a) and (b)] for a set of classical spin variables $\left\{x_{\mu}\right\}=\{+1,-1\}$ obtained from the Eq. (12) $(M=2)$. The lower panels correspond to the transition probabilities. The parameters are $q_{+}=q_{-}=1 / 2$ and $\lambda=2$.

with $\sum_{\mu=1}^{M} q_{\mu}=1$. In this case, the transition probabilities Eq. (10) can be written in terms on the number of times $n_{\mu}$ that each value $x_{\mu}$ was assumed previously,

$$
T_{n}\left(\left\{x_{i}\right\} \mid x\right)=\sum_{\mu=1}^{M} \frac{\lambda q_{\mu}+n_{\mu}}{n+\lambda} \delta\left(x-x_{\mu}\right),
$$

where $\left\{x_{i}\right\} \equiv x_{1}, \cdots x_{n}$. Notice that the set of numbers $\left\{n_{\mu}\right\}_{\mu=1}^{M}$ that the random values $\left\{X_{i}\right\}_{i=1}^{n}$ assumed the values $\left\{x_{\mu}\right\}_{\mu=1}^{M}$ satisfy the relation $n=\sum_{\mu=1}^{M} n_{\mu}$.

The correlation mechanism associated to Eq. (12) can be read in the following way. With probability $\lambda /(n+\lambda)$ the random variable $X_{n+1}$ is draw randomly in agreement with the density $P_{1}(x)$, Eq. (11). Hence, independently of the previous history, it assumes the value $x_{\mu}$ with probability $q_{\mu}$. Alternatively, with probabilities $n_{\mu} /(n+\lambda)$, which depends on all previous history, it assumes the value $x_{\mu}$. The parameter $\lambda$ measure the weigh of both options.

In order to achieve a deeper understanding of the processes defined by Eq. (12), in Fig. 1 we plotted a set of realizations for the random variables $\left\{X_{i}\right\}_{i=1}^{n}$ (upper panels). They correspond to classical spin variables, that is, we take $x_{\mu}= \pm 1$ and $M=2$. For clarity, each value of $X_{i}$ is continued in the real interval $(i-1, i)$.

The first value, $X_{1}$, is chosen in agreement with $P_{1}(x)$, Eq. (11). The next values $\left\{X_{i}\right\}_{i=2}^{n}$ follows from the transition probability $T_{n}\left(\left\{x_{i}\right\} \mid x\right)$, Eq. (12). We also plotted this object as a function of $n$ and for each value of $x= \pm 1$ (lower panels). Notice that each curve gives the probability for the next variable, given the previous history. Therefore they are random objects. We found that for increasing $n$, the transition probabilities always saturate to stationary values. Therefore, when this regime is achieved, each realization is equivalent to that of independent random variables. Nevertheless, the stationary values reached by the transition probabilities are different for each realization, that is, they are random. This property, valid for any $\lambda$, is characterized in the next Section [see Eq. (35)].

In the realization of Fig. 1(a) the stationary transition probability for the state -1 is larger than for the state +1 . Consistently, the state -1 is taken much more 

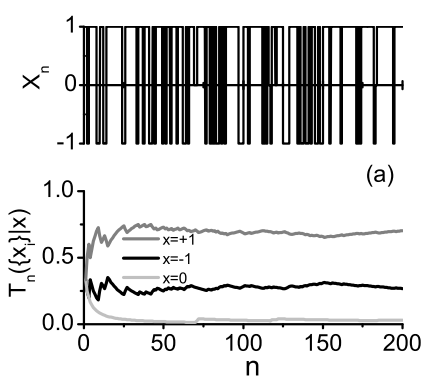

(a)

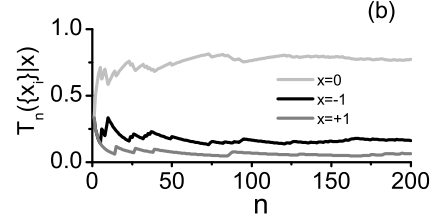

FIG. 2: Two realizations [(a) and (b)] for a set of three-state random variables $\left\{x_{\mu}\right\}=\{+1,0,-1\}$. They follows from the transition probability (12) $(M=3)$, which is plotted in the lower panels. The parameters are $q_{+}=q_{-}=q_{0}=1 / 3$ and $\lambda=3$.

frequently, feature clearly visible in the upper panel. In Fig. 1(b) the difference between the stationary values is much smaller, inducing a more "noisy" realization.

In Fig. 2 we plot a set of realizations obtained from the transition probability Eq. (12) for random variables characterized by three states, $M=3$, with $x_{\mu}=+1,0,-1$. Similarly to the case of two-level variables, for increasing $n$ the transition probabilities reach stationary values, which are different and random for each realization. Therefore, in this regime the realizations are also equivalent to that of identical independent random variables. In Fig. 2(a) the random variables almost always assume the values $x= \pm 1$. This happens because the stationary value of the transition probability corresponding the state $x=0$ is much smaller than the other two, $x= \pm 1$. Instead, in Fig. 2(b) the state $x=0$ has the larger stationary transition probability. Consistently, the states $x= \pm 1$ appear sparsely.

\section{Composed Pólya Urn scheme}

Here, we introduce a generalization of the previous urn scheme that is also consistent with interchangeability. We consider non-discrete random variables with arbitrary probability density $P_{1}(x)$. The domain $\Omega$ of each variable $\left\{X_{i}\right\}_{i=1}^{n}$, that is, the domain of $P_{1}(x)$, is split in a finite set of disjoint subdomains $\left\{\Omega_{\mu}\right\}_{\mu=1}^{M}$ such that the total domain is their union, $\Omega=\cup \Omega_{\mu}$. To each region $\Omega_{\mu}$ we associate a probability density $p_{\mu}(x)$, normalized as $\int_{\Omega} p_{\mu}(x) d x=1$. Under these definitions, we propose the transition probability density

$$
T_{n}\left(\left\{x_{i}\right\} \mid x\right)=\frac{\lambda P_{1}(x)+\sum_{\mu=1}^{M} p_{\mu}(x) \sum_{i=1}^{n} \int_{\Omega_{\mu}} d y \delta\left(y-x_{i}\right)}{n+\lambda}
$$

As before, $\lambda$ is a free parameter and $\left\{x_{i}\right\}=x_{1}, x_{2}, \cdots x_{n}$ is the previous trajectory.
The integral contributions

$$
n_{\mu} \equiv \sum_{i=1}^{n} \int_{\Omega_{\mu}} d y \delta\left(y-x_{i}\right), \quad \sum_{\mu=1}^{M} n_{\mu}=n,
$$

give the number of times the variables $\left\{x_{i}\right\}_{i=1}^{n}$ fell in the subdomain $\Omega_{\mu}$. Therefore, we can write

$$
T_{n}\left(\left\{x_{i}\right\} \mid x\right)=\frac{\lambda P_{1}(x)+\sum_{\mu=1}^{M} p_{\mu}(x) n_{\mu}}{n+\lambda} .
$$

The correlation dynamics induced by Eq. (13) is then clear. With probability $\lambda /(n+\lambda)$ the next variable, independently of the previous history, is chosen in agreement with $P_{1}(x)$. On the other hand, with probabilities $n_{\mu} /(n+\lambda)$, the next value is chosen in agreement with the arbitrary densities $p_{\mu}(x)$.

It is simple to check that the transition probability density (13), for arbitrary domains $\left\{\Omega_{\mu}\right\}_{\mu=1}^{M}$ and densities $\left\{p_{\mu}(x)\right\}_{\mu=1}^{M}$, is normalized and positive defined

$$
\int_{\Omega} d x T_{n}\left(\left\{x_{i}\right\} \mid x\right)=1, \quad T_{n}\left(\left\{x_{i}\right\} \mid x\right) \geq 0 .
$$

On the other hand, in order to be consistent with the interchangeability symmetry it must to satisfy the hierarchical relations Eq. (7). After same algebra, it follows that interchangeability is fulfilled under the condition

$$
\sum_{\mu=1}^{M} p_{\mu}(x) \int_{\Omega_{\mu}} P_{1}(y) d y=P_{1}(x)
$$

jointly with the following one,

$$
\int_{\Omega_{\mu}} p_{\mu^{\prime}}(x) d x=\delta_{\mu \mu^{\prime}}
$$

Hence, interchangeability is not fulfilled by arbitrary densities $\left\{p_{\mu}(x)\right\}$.

Condition (17) say us that the set $\left\{p_{\mu}(x)\right\}$, under appropriate weights, recover the distribution $P_{1}(x)$. Condition (18) implies that each density $p_{\mu}(x)$ is not null only on its associated subdomain $\Omega_{\mu}$. A solution to these constraints is given by

$$
p_{\mu}(x)=P_{1}(x) \frac{\theta_{\Omega_{\mu}}(x)}{\int_{\Omega_{\mu}} P_{1}\left(x^{\prime}\right) d x^{\prime}},
$$

where we defined the region indicator

$$
\theta_{\Omega_{\mu}}(x) \equiv\left\{\begin{array}{ll}
1 & \text { if } x \in \Omega_{\mu} \\
0 & \text { if } x \notin \Omega_{\mu}
\end{array} .\right.
$$

It is simple to check that (19) satisfies both constraints. Hence, interchangeability is fulfilled.

Interestingly, from the previous solutions for $\left\{p_{\mu}(x)\right\}_{\mu=1}^{M}$, Eq. (19), we can write the probability density of each variable as

$$
P_{1}(x)=\sum_{\mu=1}^{M} q_{\mu} p_{\mu}(x)
$$



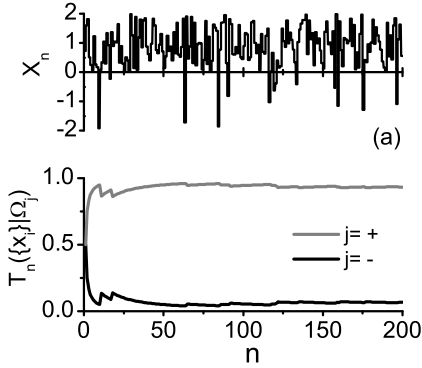

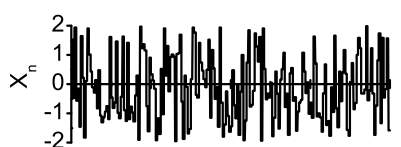

(b)

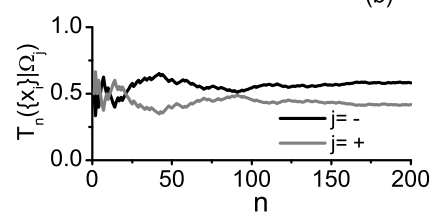

FIG. 3: Two realizations [(a) and (b)] of the composed Pólya urn scheme [Eq. (23)] defined by the Eqs. (24) and (25). The lower panels correspond to the transition probabilities associated to the subdomains $\Omega_{+}$and $\Omega_{-}$, each one having weights $q_{+}=q_{-}=1 / 2$ (see text). The parameter is $\lambda=2$.

where the positive weights are

$$
q_{\mu} \equiv \int_{\Omega_{\mu}} P_{1}(x) d x
$$

and fulfill $\sum_{\mu=1}^{M} q_{\mu}=1$. In consequence, by using Eq. (15), the transition probability Eq. (13) reads

$$
T_{n}\left(\left\{x_{i}\right\} \mid x\right)=\sum_{\mu=1}^{M} \frac{\lambda q_{\mu}+n_{\mu}}{n+\lambda} p_{\mu}(x) .
$$

This final expression is the main result of this section.

Eq. (23) has a stretched relation with the standard Pólya urn scheme, Eq. (12). In fact, both expressions are related by the replacements $\delta\left(x-x_{\mu}\right) \leftrightarrow p_{\mu}(x)$. On the other hand, the weights in the single density Eq. (11) here follows from Eqs. (21) and (22). Hence, each subdomain $\Omega_{\mu}$ can be associated to the states $x_{\mu}$ [Eq. (11)]. Nevertheless, instead of the value $x_{\mu}$, here the next variable assumes a random value distributed over the subdomain $\Omega_{\mu}$ with probability density $p_{\mu}(x)$. In consequence, Eq. (23) can be read as an independent statistical composition of the Pólya urn scheme, Eq. (12), and the set of probability densities $\left\{p_{\mu}(x)\right\}_{\mu=1}^{M}$.

As an example, we take the uniform probability density

$$
P_{1}(x)=\frac{1}{4}, \quad-2 \leq x \leq 2,
$$

and $P_{1}(x)=0$ if $x \notin[-2,2]$. Therefore, each variable only assume random values over the real interval $[-2,2]$. Then $\Omega=\{x \in[-2,2]\}$. The composed urn scheme is completely characterized after defining the subdomains $\left\{\Omega_{\mu}\right\}$. We consider only two subspaces, $\Omega_{+}$and $\Omega_{-}$, defined as $\Omega_{+}=\{x \in[0,2]\}$ and $\Omega_{-}=\{x \in[-2,0)\}$ respectively. Notice that $\Omega=\Omega_{+} \cup \Omega_{-}$. The associated probability densities, from Eqs. (19) and (20) becomes

$$
\begin{array}{ll}
p_{+}(x)=\frac{1}{2}, & 0 \leq x \leq 2, \\
p_{-}(x)=\frac{1}{2}, & -2 \leq x<0 .
\end{array}
$$

Notice that the underlying discrete process that decides which probability density is chosen, $p_{+}(x)$ or $p_{-}(x)$, is equivalent to that plotted in Fig. 1. In fact, from Eq. (22) if follows $q_{+}=q_{-}=1 / 2$.

In Fig. 3 we plot a set of realizations corresponding to the previous definitions. In contrast to the previous figures, here the random variables assume values over the real interval $[-2,2]$. In the lower panels we plot the underlying transition probability $T_{n}\left(\left\{x_{i}\right\} \mid \Omega_{j}\right)$ with governs which subdomain $(j= \pm)$ is occupied in the next step. Consistently, its behavior is similar to that of Fig. 1. In Fig. 3(a) the subspace $\Omega_{+}$has a higher stationary probability and, consistently, the realization take most of its values in the interval $[0,2]$. In Fig. 3(b) both subspaces have similar stationary values. Hence, the realization looks like a random signal in the full domain $[-2,2]$. On the other hand, by averaging over realizations we checked that the probability density of each variable $\left\{X_{i}\right\}_{i=1}^{n}$ is given by $P_{1}(x)$, Eq. (24).

\section{STATISTICS OF THE SUM VARIABLE}

In the previous Section (and in the Appendixes) we described different memory mechanism and statistics consistent with the interchangeability property. Here, we study departures with respect to the standard CLT when considering such kind of globally correlated variables.

We consider the normalized random sum variable

$$
W=\lim _{n \rightarrow \infty} W_{n}=\lim _{n \rightarrow \infty}\left(\frac{1}{n} \sum_{i=1}^{n} X_{i}\right) .
$$

Notice that in contrast with the standard CLT [1-4] , instead of $1 / \sqrt{n}$, here the normalization is $1 / n$. We choose this factor because all studied models, depending on their characteristic parameters, are able to reach a full correlated regime where all variables $\left\{X_{i}\right\}$ assume the same random value. Hence, in that regime the normalization $1 / n$ is the only one that delivers a random variable $(W)$ that (asymptotically) does not depend on $n$.

The probability density $P(w)$ of $W$ can be written as the following limit, $P(w)=\lim _{n \rightarrow \infty} P\left(w_{n}\right)$,

$$
P(w)=\lim _{n \rightarrow \infty} \int d x_{1} \cdots d x_{n} \delta\left(w-\frac{1}{n} \sum_{i=1}^{n} x_{i}\right) P_{n}\left(\left\{x_{i}\right\}\right),
$$

where $P_{n}\left(\left\{x_{i}\right\}\right) \equiv P_{n}\left(x_{1}, \cdots x_{n}\right)$ is the $n$-joint probability density. By introducing the Fourier representation of the delta Dirac function, $\delta(x)=(1 / 2 \pi) \int_{-\infty}^{+\infty} e^{-i k x} d k$, the characteristic function $G_{w}(k)$ of $P(w)$,

$$
G_{w}(k)=\int_{-\infty}^{+\infty} d w e^{i k w} P(w),
$$

can be written as, $G_{w}(k)=\lim _{n \rightarrow \infty} G_{w_{n}}(k)$,

$$
G_{w}(k)=\lim _{n \rightarrow \infty} \int d x_{1} \cdots d x_{n} \exp \left(i \frac{k}{n} \sum_{i=1}^{n} x_{i}\right) P_{n}\left(\left\{x_{i}\right\}\right) .
$$


In terms of the the multiple Fourier transform of $P_{n}\left(\left\{x_{i}\right\}\right)$, that is,

$$
G_{n}\left(\left\{k_{i}\right\}\right)=\int d x_{1} \cdots d x_{n} \exp \left(i \sum_{i=1}^{n} k_{i} x_{i}\right) P_{n}\left(\left\{x_{i}\right\}\right),
$$

it follows

$$
G_{w}(k)=\lim _{n \rightarrow \infty} G_{w_{n}}(k)=\lim _{n \rightarrow \infty} G_{n}\left(\left\{k_{i}=\frac{k}{n}\right\}\right) .
$$

Below we treat the different cases introduced previously. For clarifying the derivation of some results, the well known case of independent variables is reviewed first.

\section{A. Statistical independent variables}

Assume the set $\left\{X_{i}\right\}_{i=1}^{n}$ are independent random variables with probability density distribution $P_{1}(x)$. Therefore, $P_{n}\left(\left\{x_{i}\right\}\right)=\prod_{i=1}^{n} P_{1}\left(x_{i}\right)$. From Eq. (28), it follows

$$
G_{w_{n}}(k)=\left[G_{x}\left(\frac{k}{n}\right)\right]^{n},
$$

where $G_{x}(k)$ is the Fourier transform of $P_{1}(x)$.

For small $k$, we assume valid the approximation $G_{x}(k) \simeq e^{i k\langle x\rangle}\left(1-\frac{\sigma^{2} k^{2}}{2}\right)$, where

$$
\bar{x}=\int_{-\infty}^{+\infty} d x x P_{1}(x), \quad \sigma^{2}=\int_{-\infty}^{+\infty} d x(x-\bar{x})^{2} P_{1}(x),
$$

are the mean value and standard deviation of the distribution $P_{1}(x)$. Therefore, we can approximate $G_{w_{n}}(k) \simeq$ $e^{i k \bar{x}}\left[1-\frac{\sigma^{2} k^{2}}{2 n^{2}}\right]^{n}$, which can be rewritten as

$$
G_{w_{n}}(k) \simeq e^{i k \bar{x}} \exp \left[-\frac{1}{2} \frac{\sigma^{2} k^{2}}{n}\right] .
$$

After Fourier inversion it follows

$$
P\left(w_{n}\right) \simeq \sqrt{\frac{1}{2 \pi\left(\sigma^{2} / n\right)}} \exp \left[-\frac{1}{2} \frac{\left(w_{n}-\bar{x}\right)^{n}}{\left(\sigma^{2} / n\right)}\right],
$$

which is a Gaussian distribution. Given that $P(w)=$ $\lim _{n \rightarrow \infty} P\left(w_{n}\right)$, it follows that

$$
P(w)=\delta(w-\bar{x})
$$

Therefore, the random variable $W$ deterministically assume the value $\bar{x}$. This result, which can be read as the well known law of large numbers [1-4], follows from the normalization $1 / n$ in Eq. (26). In fact, defining the variable $\sqrt{n} W$ from Eq. (33) one recovers a Gaussian distribution that does not depends on $n$, which in turn corresponds to the standard CLT. The basin or domain of attraction of the normal distribution corresponds to all distributions $P_{1}(x)$ with finite first and second moments.

Using the same Fourier techniques, we showed that departure with respect to Eq. (34) arise from (correlated) Gaussian variables [see Eq. [B16)] and also in the de Finetti representation [see Eq. (C5)]. In fact, the possibility of achieving a fully correlated regime is enough for warranting departure from a delta distribution.

\section{B. Pólya Urn scheme}

In the previous section, we explicitly showed a very important property of the Pólya urn scheme, that is, for increasing $n$ the transition probabilities converge to that of identical independent random variables. Nevertheless, the stationary values achieved by the transition probability are random, that is, their are different for each realization. This property was characterized previously in the literature [27, 28]. Here, we review it in order to characterize the sum variable (26).

The transition probability Eq. (12), in the asymptotic regime is characterized by the weights

$$
F_{\mu} \equiv \lim _{n \rightarrow \infty} \frac{\lambda q_{\mu}+n_{\mu}}{n+\lambda}, \quad 0 \leq F_{\mu} \leq 1,
$$

which consistently satisfy $\sum_{\mu=1}^{M} F_{\mu}=1$. These weights (probabilities) are different for each realization, that is, their are random variables. Hence, taking an ensemble of realizations [see Figs. (1) and (2)] one can define their probability density $D\left(\left\{f_{\mu}\right\} \mid\left\{\lambda_{\mu}\right\}\right)$, which depends on the characteristic parameters of the problem, here defined as

$$
\lambda_{\mu} \equiv \lambda q_{\mu} .
$$

Due to the normalization of the weights $\left\{q_{\mu}\right\}_{\mu=1}^{M}$, it follows $\lambda=\sum_{\mu=1}^{M} \lambda_{\mu}$. It is known that $D\left(\left\{f_{\mu}\right\} \mid\left\{\lambda_{\mu}\right\}\right)$ is a Dirichlet distribution [27, 28],

$$
D\left(\left\{f_{\mu}\right\} \mid\left\{\lambda_{\mu}\right\}\right) \equiv \frac{\Gamma(\lambda)}{\prod_{\mu^{\prime}=1}^{M} \Gamma\left(\lambda_{\mu^{\prime}}\right)} \prod_{\mu=1}^{M} f_{\mu}^{\lambda_{\mu}-1},
$$

where $\Gamma(x)$ is the Gamma function. $D\left(\left\{f_{\mu}\right\} \mid\left\{\lambda_{\mu}\right\}\right)$ is positive for all values of $\left\{f_{\mu}\right\}_{\mu=1}^{M}$, and normalized as $\int_{\Lambda} d f_{1} \cdots d f_{M-1} D\left(\left\{f_{\mu}\right\} \mid\left\{\lambda_{\mu}\right\}\right)=1$, where $\Lambda$ is the region defined by $\sum_{\mu=1}^{M} f_{\mu}=1$. On the other hand, the relation $q_{\nu}=\int_{\Lambda} d f_{1} \cdots d f_{M-1} D\left(\left\{f_{\mu}\right\} \mid\left\{\lambda_{\mu}\right\}\right) f_{\nu}$ is fulfilled for all $\nu=1, \cdots M$.

From Eq. (37) we can obtain the probability density $P(w)$ of the variable $W$. Given that asymptotically each realization is equivalent to that of independent random variables, one can associate the probability density $\delta\left(w-\bar{X}_{f}\right)$ to each realization [see Eq. (34)], where $\bar{X}_{f}=\sum_{\mu=1}^{M} F_{\mu} x_{\mu}$. Now, the final structure of $P(w)$ arises after averaging over realizations. Given that the random variables $F_{\mu}$ obeys the statistics given by Eq. (37), it follows

$$
P(w)=\int_{\Lambda} d f_{1} \cdots d f_{M-1} \delta\left(w-\bar{x}_{f}\right) D\left(\left\{f_{\mu}\right\} \mid\left\{\lambda_{\mu}\right\}\right),
$$

where

$$
\bar{x}_{f} \equiv \sum_{\mu=1}^{M} f_{\mu} x_{\mu} .
$$

From the result Eq. (38), in the limit of $\lambda \rightarrow \infty$ consistently we recover the independent random variables case, 

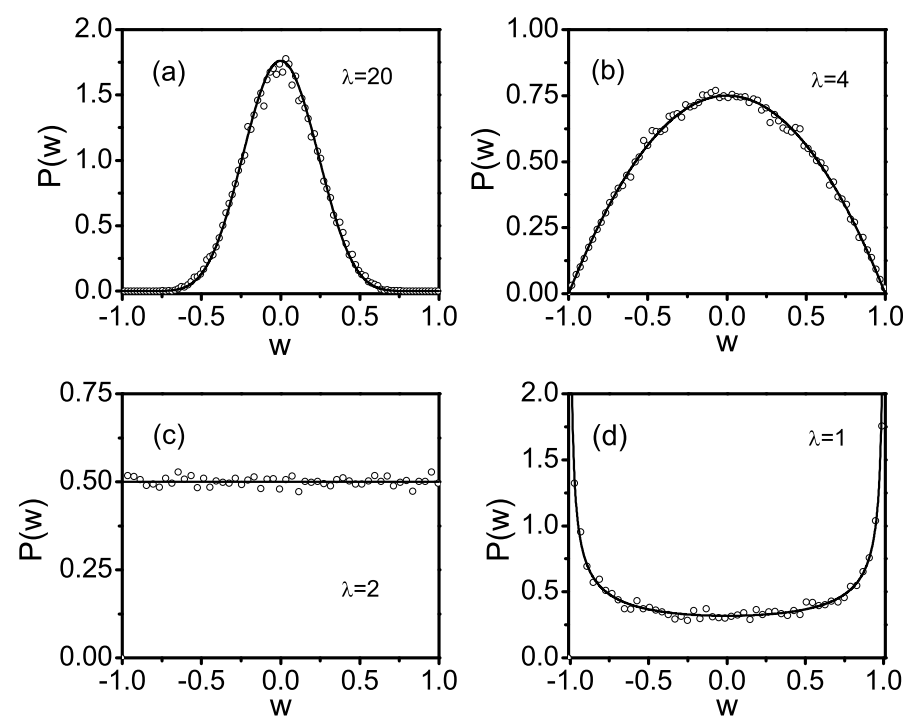

FIG. 4: Probability density of the sum variable $W$, Eq. (26). The random contributions are classical spin variables, $x_{\mu}= \pm 1$, defined by the transtion probability (12). The full lines correspond to the analytical expression Eq. (40), while the circles correspond to numerical simulations with $n=300$ terms and $5 \times 10^{5}$ realizations (see Fig. 1). In all cases, $q_{+}=q_{-}=1 / 2$.

$\lim _{\lambda \rightarrow \infty} P(w)=\delta\left(w-\bar{x}_{q}\right)$, where $\bar{x}_{q} \equiv \sum_{\mu=1}^{M} q_{\mu} x_{\mu}$. In the limit $\lambda \rightarrow 0$, which corresponds to the fully correlated case, it follows $\lim _{\lambda \rightarrow 0} P(w)=\sum_{\mu=1}^{M} q_{\mu} \delta\left(w-x_{\mu}\right)$ [see Eq. (12)].

The final expression (38) allow us to characterize the CLT for the Pólya urn scheme. It is valid for any value of $M$ and arbitrary discrete distributions, Eq. (11). For example, for classical spin variables, $x_{\mu}= \pm 1$, after integration we get $\left(\lambda_{ \pm} \equiv \lambda q_{ \pm}\right)$

$$
P(w)=\frac{1}{\mathcal{N}}(1+w)^{\lambda_{+}-1}(1-w)^{\lambda_{-}-1},
$$

where $\mathcal{N} \equiv 2^{\lambda_{+}+\lambda_{-}-1} \Gamma\left(\lambda_{+}\right) \Gamma\left(\lambda_{-}\right) / \Gamma\left(\lambda_{+}+\lambda_{-}\right)$.

We notice that Eq. (40) can be read as a Beta [2] or asymmetric $q$-Gaussian distribution [20]. In the symmetric case $\lambda_{+}=\lambda_{-}$, this result was derived previously in the context of a nonextensive thermodynamics approach [13] (see also [2, 26]).

In Fig. 4 we obtained numerically $P(w)$ by averaging a set of realizations such as those presented in Fig. 1. Results for different values of $\lambda$ are presented, while $q_{+}=$ $q_{-}=1 / 2$. Independently of the parameter values, we find that Eq. (40) fits the numerical results.

For three-states variables with $\left\{x_{\mu}\right\}=\{+1,0,-1\}$, the parameters are $\left\{q_{\mu}\right\}=\left\{q_{+}, q_{0}, q_{-}\right\}$and $\lambda$. They can be parametrized as $\left\{\lambda_{\mu}\right\}=\left\{\lambda q_{\mu}\right\}=\left\{\lambda_{+}, \lambda_{0}, \lambda_{-}\right\}$. By taking into account that $\bar{x}_{f}=f_{+}-f_{-}$[Eq. (39)], from Eq. (38) we get,

$$
P(w)= \begin{cases}g_{+}[w] & w>0 \\ g_{-}[w] & w<0\end{cases}
$$
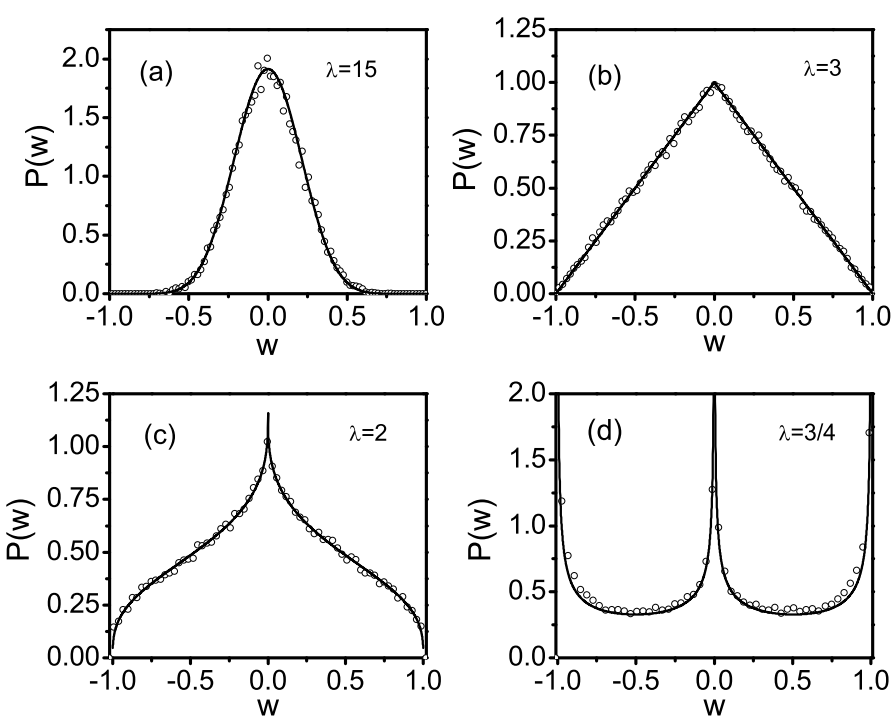

FIG. 5: Probability density $P(w)$ for random variables with three discrete states, $x_{\mu}=+1,0,-1$, obtained from the transtion probability (12). The full lines correspond to the analytical expression Eq. (41), while the circles correspond to a numerical simulation with $n=300$ terms and $5 \times 10^{5}$ realizations (see Fig. 2). In all cases, $q_{+}=q_{0}=q_{-}=1 / 3$.

where each contribution is defined as

$g_{+}[w]=\int_{0}^{\frac{1-w}{2}} d f f^{\lambda_{-}-1}(1-w-2 f)^{\lambda_{0}-1}(f+w)^{\lambda_{+}-1}$,
$g_{-}[w]=\int_{0}^{\frac{1+w}{2}} d f(f-w)^{\lambda_{-}-1}(1+w-2 f)^{\lambda_{0}-1} f^{\lambda_{+}-1}$.

These integrals can be solved in terms of the hypergeometric function ${ }_{2} F_{1}[a, b, c, z]$ as

$$
\begin{aligned}
g_{+}[w]= & \frac{(1-w)^{\lambda_{-}+\lambda_{0}-1} w^{\lambda_{+}-1}}{2^{\lambda_{-}} \Gamma^{-1}(\lambda) \Gamma\left(\lambda_{+}\right) \Gamma\left(\lambda_{0}+\lambda_{-}\right)} \\
& { }_{2} F_{1}\left[\lambda_{-}, 1-\lambda_{+}, \lambda_{0}+\lambda_{-}, \frac{w-1}{2 w}\right],
\end{aligned}
$$

and similarly

$$
\begin{aligned}
g_{-}[w]= & \frac{(1+w)^{\lambda_{+}+\lambda_{0}-1}(-w)^{\lambda_{-}-1}}{2^{\lambda_{+}} \Gamma^{-1}(\lambda) \Gamma\left(\lambda_{-}\right) \Gamma\left(\lambda_{0}+\lambda_{+}\right)} \\
& { }_{2} F_{1}\left[1-\lambda_{-}, \lambda_{+}, \lambda_{0}+\lambda_{+}, \frac{w+1}{2 w}\right] .
\end{aligned}
$$

The hypergeometric function is defined by ${ }_{2} F_{1}[a, b, c, z]=$ $\sum_{k=0}^{\infty}(a)_{k}(b)_{k}(c)_{k} z^{k} / k !$ with $(x)_{k}=\prod_{j=0}^{k}(x+j)$. Simpler expressions can be found in the particular case $\lambda_{+}=$ $\lambda_{-1}=1$, where Eq. (41) reduces to

$$
P(w)=\frac{1}{2}\left(1+\lambda_{0}\right)(1-|w|)^{\lambda_{0}}, \quad \lambda_{+}=\lambda_{-1}=1 .
$$

In Fig. 5 we show a set of plots corresponding to $P(w)$. The realizations, over which the distribution are 
obtained, are those shown in Fig. 2. We found that the density (41) fits the numerical results. The case shown Fig. 5(b) corresponds to Eq. (42) with $\lambda_{0}=1$.

\section{Composed Pólya Urn scheme}

The previous results with the Pólya urn scheme (see also the Appendixes) demonstrates that the sum variable Eq. (26), depending on the underlying correlation mechanism, may adopt very different statistics. In contrast to independent random variables, these probabilities do not have associated a basin of attraction. Here, we show that the composed Pólya urn scheme fall in the basin of attraction of the standard scheme. This is the main result of this section.

For the composed Pólya urn scheme, the probability density of the sum variable $W$ [Eq. (26)] is given by Eq. (38) with $\left\{q_{\mu}\right\}_{\mu=1}^{M}$ given by Eq. (22) and under the replacement

$$
\bar{x}_{f}=\sum_{\mu=1}^{M} f_{\mu} \bar{x}_{\mu}, \quad \bar{x}_{\mu}=\int_{\Omega_{\mu}} d x x p_{\mu}(x) .
$$

Therefore, the main change corresponds to $x_{\mu} \rightarrow \bar{x}_{\mu}$ [Eq. (39)] where $\bar{x}_{\mu}$ is the mean value associated to the distribution $p_{\mu}(x)$. This result say us that all random variables obtained from the composed Pólya urn scheme are in the basin of the attractors corresponding to the standard urn scheme, Eq. (38). As shown below, this result relies on the applicability of the law of large numbers to random independent variables draw randomly from any of the distributions $\left\{p_{\mu}(x)\right\}_{\mu=1}^{M}$.

For demonstrating the previous result we use that the composed Pólya urn scheme consist of two independent random processes: the randomness introduced by the probability densities $p_{\mu}(x)$ associated to each subdomain $\Omega_{\mu}$ and the underlying Pólya urn process that select each subdomain. Therefore, the joint probability density of the random variables $\left\{X_{i}\right\}_{i=1}^{n}$ reads

$$
P_{n}\left(\left\{x_{i}\right\}\right)=\left\langle p_{\mu_{1}}\left(x_{1}\right) \cdots p_{\mu_{n}}\left(x_{n}\right)\right\rangle_{\{\mu\}} .
$$

Here, each index $\mu_{i}=1 \cdots M$ runs over the set of subdomains $\left\{\Omega_{\mu}\right\}_{\mu=1}^{M}$. On other hand, $\langle\cdots\rangle_{\{\mu\}}$ denotes and average over the ensemble of realizations associated to the underlying Pólya urn scheme. From Eqs. (28) and (29) we get $G_{w}(k)=\lim _{n \rightarrow \infty} G_{w}^{(n)}(k)$, with

$$
G_{w}^{(n)}(k)=\left\langle G_{\mu_{1}}\left(\frac{k}{n}\right) \cdots G_{\mu_{n}}\left(\frac{k}{n}\right)\right\rangle_{\{\mu\}},
$$

where $G_{\mu}(k)$ is the Fourier transform of $p_{\mu}(x)$. By indexing the realizations by the number of times $n_{\mu}$ that each subspace $\Omega_{\mu}$ is selected, we can write

$$
G_{w}^{(n)}(k)=\left\langle\left[G_{1}\left(\frac{k}{n}\right)\right]^{n_{1}} \cdots\left[G_{M}\left(\frac{k}{n}\right)\right]^{n_{M}}\right\rangle_{\{n\}} .
$$

Taking into account that when $n \rightarrow \infty$ the set of occurrences also diverge, $\left\{n_{\mu}\right\} \rightarrow \infty$, each factor in the previous expression can be approximated as

$$
\left[G_{\mu}\left(\frac{k}{n}\right)\right]^{n_{\mu}} \approx \exp \left(i k \bar{x}_{\mu} \frac{n_{\mu}}{n}\right) \exp \left(-\frac{\sigma_{\mu}^{2} k^{2}}{2 n} \frac{n_{\mu}}{n}\right),
$$

where $\bar{x}_{\mu}$ is the mean value defined in Eq. (43) while $\sigma_{\mu}^{2}=\int_{\Omega_{\mu}} d x\left(x-\bar{x}_{\mu}\right)^{2} p_{\mu}(x)$. Notice that the previous approximation is equivalent to the validity of the law of large numbers for each density $p_{\mu}(x)$ [see Eq. (32)].

In the previous approximation, the argument $n_{u} / n$, in the asymptotic limit, can be associated with the random variables $F_{\mu}$, Eq. (35). Therefore $n_{u} / n \simeq F_{\mu}$, which from Eqs. (46) and (47) lead to

$$
G_{w}(k)=\left\langle\exp i k \sum_{\mu} F_{\mu} \bar{x}_{\mu}\right\rangle_{\{F\}} .
$$

The average over the random set of weights $\{F\}$ is governed by the Dirichlet distribution Eq. (37). Therefore, after Fourier inversion we recover Eq. (38), where instead of Eq. (39), now it applies Eq. (43). This finish the demonstration.

As an example of the previous result we take a composed Pólya urn scheme [Eq. (23)] defined with two subdomains $\Omega_{ \pm}$with densities $p_{ \pm}(x)$. We get [Eq. (46)]

$$
G_{w}^{(n)}(k)=\left\langle\left[G_{+}\left(\frac{k}{n}\right)\right]^{n+}\left[G_{-}\left(\frac{k}{n}\right)\right]^{n_{-}}\right\rangle_{\{n\}},
$$

where $n_{ \pm}$are the number of times that each subspace $\Omega_{ \pm}$was chosen, and $G_{ \pm}(k)=\int_{-\infty}^{+\infty} d w e^{i k w} p_{ \pm}(w)$. Using that $n_{+}+n_{-}=n$, it follows

$$
G_{w}^{(n)}(k)=\sum_{n_{+}=0}^{n} P_{n}\left(n_{+}\right)\left[G_{+}\left(\frac{k}{n}\right)\right]^{n_{+}}\left[G_{-}\left(\frac{k}{n}\right)\right]^{n-n_{+}}
$$

where $P_{n}\left(n_{+}\right)$is the probability of the random variable $n_{+}$. This object, after some algebra and by using the properties of Gamma functions, can be obtained from from Eqs. (4) and (12). Alternatively, it can be obtained directly from de Finetti representation theorem [see. Eq. (C12)]. It reads

$$
P_{n}\left(n_{+}\right)=\frac{1}{\mathcal{N}_{n}}\left(\begin{array}{c}
n \\
n_{+}
\end{array}\right) \frac{\Gamma\left(n_{+}+\lambda_{+}\right)}{\Gamma\left(\lambda_{+}\right)} \frac{\Gamma\left(n-n_{+}+\lambda_{-}\right)}{\Gamma\left(\lambda_{-}\right)}
$$

where $\mathcal{N}_{n}=\Gamma(n+\lambda) / \Gamma(\lambda)$, and $\lambda_{ \pm}=\lambda q_{ \pm}$[Eq. (22) $]$.

The previous two expressions give an exact analytical expression for $G_{w}^{(n)}(k)$. For the example defined by Eq. (24), the random variables have a uniform distribution for $X \in[-2,2]$. The probabilities of each subdomain are defined by Eq. (25). Their Fourier transform read

$$
G_{ \pm}(k)=[\sin (k) / k] e^{ \pm i k} .
$$

In order to check these results, in Fig. 6 we show a set of probability distributions obtained by averaging the realizations of the composed scheme (Fig. 3). For each 

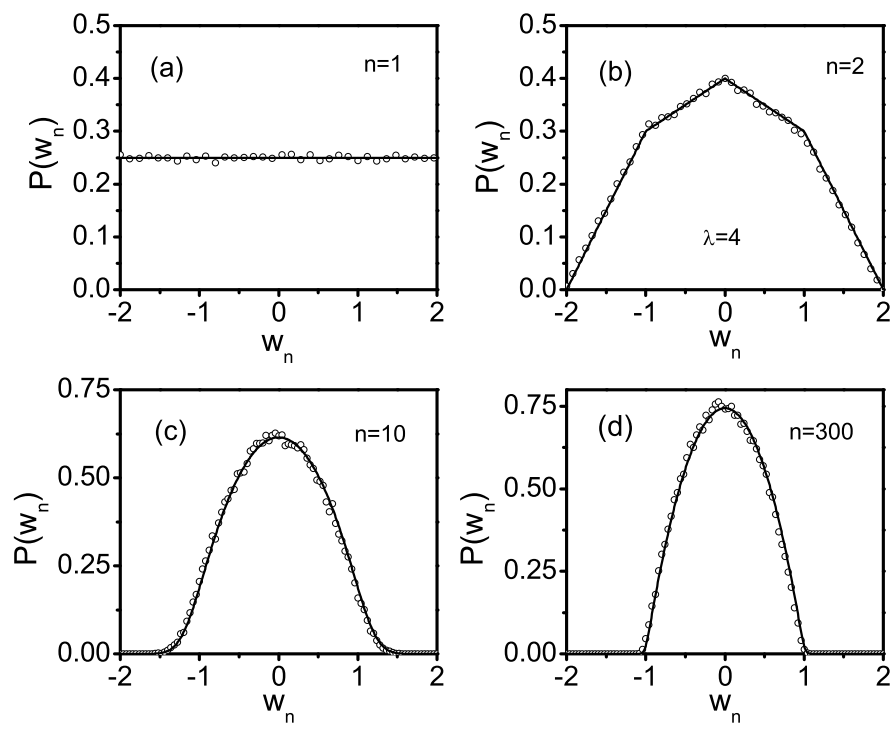

FIG. 6: Probability density $P\left(w_{n}\right)$ of the variable $W_{n}=$ $(1 / n) \sum_{i=1}^{n} X_{i}$, where each random variable $X_{i}$ follows from the composed Pólya urn scheme defined by Eqs. (23) and (24), with $\lambda=4$. The weights [Eq. (22)] are $q_{+}=q_{-}=1 / 2$. The solid line follows from the inverse Fourier transform of Eq. (50) defined with Eq. (52). The circles correspond to numerical results obtained by averaging $5 \times 10^{5}$ realizations.

$n=1,2,10,300$, the numerical results follows after averaging $5 \times 10^{5}$ realizations. For $n=1$ it is recovered Eq. (24). For higher $n$ we find that the (numerical) inverse Fourier transform of Eq. (50) evaluated with Eq. (52) fits very well the numerical results (circles). Consistently with the previous analysis, at $n=300$ the density $P\left(w_{n}\right)$ is almost indistinguishable from the corresponding attractor, that is, $P\left(w_{n}\right)$ in Fig. 6(d) is very well fitted by the density $P(w)$ corresponding to the standard scheme, Eq. (40), which in turn is plotted in Fig. 4(b). This follows because the average values $\left\{\bar{x}_{\mu}\right\}$ [Eq. (43)] are $\bar{x}_{ \pm}= \pm 1$ and also the weights $\left\{q_{\mu}\right\}$ [Eq. (22)] are $q_{ \pm}=1 / 2$, which correspond to the parameters of Fig. 4. We also checked that for all values of $\lambda$ the attractors correspond to those shown in that figure.

For arbitrary distributions $P_{1}(x)$ the sum variable, associated to the composed urn scheme with two subdomains, is characterized by the attractor Eq. (40). In general, the random variables can assume values over the entire real line. For example, we take

$$
P_{1}(x)=(1 / 2) \exp (-|x|),
$$

with subdomains $\Omega_{ \pm}=\{x \lessgtr 0\}$. Then, the Fourier transforms of $p_{ \pm}(x)=\exp (\mp x)$ read

$$
G_{ \pm}(k)=\frac{1}{1 \mp i k} .
$$

In Fig. 7 we show a set of probability distributions for the sum variable for this alternative single statistics. As in the previous case, the analytical expressions in the
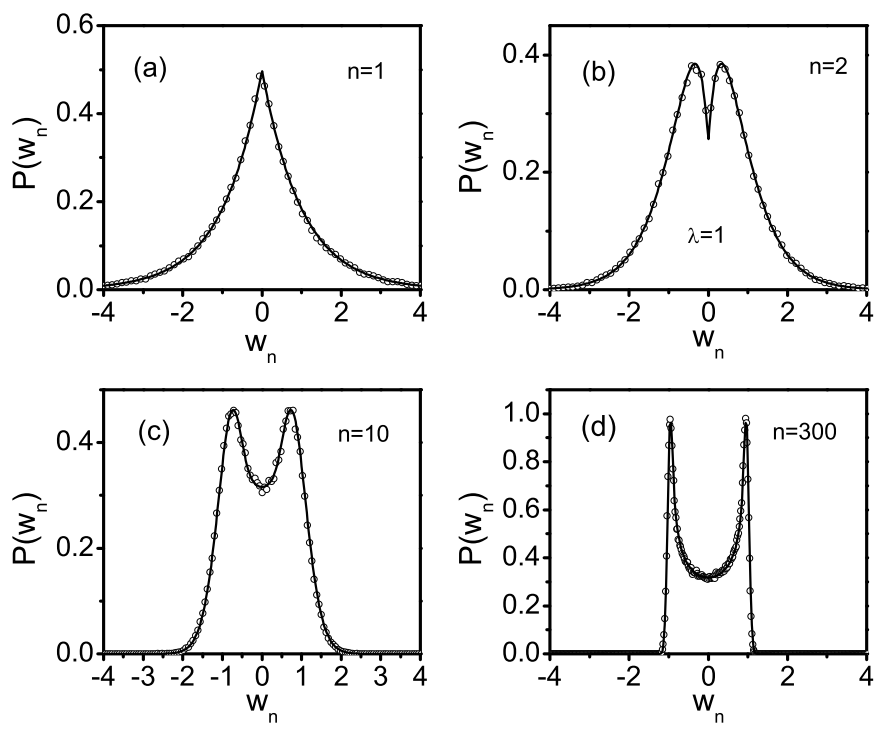

FIG. 7: Probability density $P\left(w_{n}\right)$ of the variable $W_{n}=$ $(1 / n) \sum_{i=1}^{n} X_{i}$, where each random variable $X_{i}$ follows from the composed Pólya urn scheme defined by Eqs. (23) and (53), with $\lambda=1$. The weights [Eq. (22)] are $q_{+}=q_{-}=1 / 2$. The solid line follows from the inverse Fourier transform of Eq. (50) defined with Eq. (54). The circles correspond to numerical results obtained by averaging $5 \times 10^{5}$ realizations.

Fourier domain fit the numerical results. Notice that even when the single variables assume values over the real line, their normalized sum is characterized by an (probability density) attractor that is not null only in the interval $(-1,1)$ [see Fig. 4(d)]. This property is induced by the global correlation effects.

For a urn model with three states similar results can be obtained. For example, by maintaining $P_{1}(x)$ given by Eq. (24), taking the subdomains $\Omega_{+}=\{x \in(1 / 3,5 / 3)\}$, $\Omega_{-}=\{x \in(-5 / 3,-1 / 3)\}$, and $\Omega_{0}=\{x \in(-2,-5 / 3) \cup$ $(5 / 3,2)\}$ lead to the attractors shown in Fig. 5. A model with exponential distributed variables can also be written.

\section{SUMMARY AND CONCLUSIONS}

Beyond statistically independent variables, there exist very few generalizations of the CLT. Here, we studied this problem for globally correlated random variables that are similar and interchangeable. In order to characterize these symmetries we derived a hierarchical set of equations that the transition probability densities must to satisfy, Eq. (77). These integral equations provide a tool for constructing correlation mechanisms that satisfy the required properties.

Different correlations mechanisms lead to transitions probability densities that fulfill the demanded symmetries, such as globally correlated Gaussian variables, de Finetti representation (see Appendixes) and urn schemes. 
In this last context, we introduced a generalization of Pólya urn scheme, where the values assumed by the random variables are split in different subdomains over the real line, each one being endowed with a probability density. Each subdomain is chosen in agreement with the standard Pólya urn scheme, while the associated probability density delivers the next random value (Fig. 3). The transition probability of this composed scheme, Eq. (23), fulfill the required symmetries.

The sum variable that define the CLT, Eq. (26), here is defined with a different normalization because the studied random variables may achieve a fully correlated regime. Thus, the case of independent variables leads to a delta Dirac distribution, fact related with the validity of the law of large numbers. In general, global correlations consistent with the demanded symmetries lead to very different statistics of the sum variable. The Pólya urn scheme, depending on its number of states and characteristic parameters, delivers different probability densities, Eq. (38) (see Figs. 4 and 5). For two states, the attractor is defined by an asymmetric $q$-Gaussian density $(q<1)$, Eq. (40). More complex expressions arise for more states.

Given the diversity of possible attractors, a very difficult task is to define their basin of attraction. We solved this problem in a constructive way. We demonstrated that sum of random variables generated via the composed Pólya urn scheme are in the basin of attraction of the distributions associated to the standard Pólya urn scheme (see Figs. 6 and 7). This basin is as wide as in the standard CLT. In fact, there exist infinite single probability distributions that with a specific splitting of their domain lead to the same attractor [see Eqs. (21) and (22)]. The mechanism that guarantees this result is the validity of the law of large numbers for the probability density of each subdomain as well as the convergence to stationary values of the transition probability of the standard Pólya urn scheme.

The basin of attraction of the Pólya urn attractors can be extended after raising up the interchangeability symmetry in the composed scheme [Eqs. (17) and (18)]. In addition, the same attractors arise, for example, by introducing correlations between the random variables in such a way that the law of large numbers remains valid in each subdomain. On the other hand, the present results lead us to ask about physical systems characterized by dynamical correlations able to induce attractors that take values on a subdomain of the underlying random process (variables).

In conclusions, we developed a consistent approach for dealing with globally correlated similar interchangeable random variables, which in turn allowed us to characterize different attractors of the CLT as well as their basin of attraction.

\section{Acknowledgments}

This work was supported by Consejo Nacional de Investigaciones Científicas y Técnicas (CONICET), Argentina.

\section{Appendix A: Interchangeability condition for the conditional probabilities}

Here, we derive the hierarchical set of conditions defined by Eq. (77). Assuming that interchangeability is valid for $P_{n}\left(x_{1}, \cdots x_{n}\right)$, we determine the conditions under which $P_{n+1}\left(x_{1}, \cdots x_{n+1}\right)$ also fulfill the symmetry. These functions are related as $P_{n+1}\left(x_{1}, \cdots x_{n+1}\right)=P_{n}\left(x_{1}, \cdots x_{n}\right) T_{n}\left(x_{1}, \cdots x_{n} \mid x_{n+1}\right)$. Therefore, $T_{n}\left(x_{1}, \cdots x_{n} \mid x_{n+1}\right)$ must also be symmetric in the $x_{1}, \cdots x_{n}$ arguments. The interchangeability for $P_{n+1}\left(x_{1}, \cdots x_{n+1}\right)$ is valid when $x_{n+1}$ can be interchanged with an arbitrary $x_{k}$, with $k=1, \cdots n$. Written in an explicit way, this requirement reads

$$
P_{n+1}\left(x_{1}, \cdots, x_{k}, \cdots x_{n+1}\right)=P_{n+1}\left(x_{1}, \cdots, x_{n+1}, \cdots x_{k}\right) .
$$

By using Bayes rule, these objects can be written as

$$
\begin{array}{r}
P_{n+1}\left(x_{1}, \cdots, x_{k}, \cdots x_{n+1}\right)=P_{k-1}\left(x_{1} \cdots x_{k-1}\right) \\
\times T_{k-1}\left(x_{1} \cdots x_{k-1} \mid x_{k}\right) \\
\times T_{k}\left(x_{1}, \cdots x_{k} \mid x_{k+1}\right) \\
\times T_{k+1}\left(x_{1}, \cdots, x_{k}, x_{k+1} \mid x_{k+2}\right) \\
\cdots \times T_{n-1}\left(x_{1}, \cdots x_{n-1} \mid x_{n}\right) \\
\times T_{n}\left(x_{1}, \cdots x_{n} \mid x_{n+1}\right),
\end{array}
$$

and also

$$
\begin{array}{r}
P_{n+1}\left(x_{1}, \cdots, x_{n+1}, \cdots x_{k}\right)=P_{k-1}\left(x_{1} \cdots x_{k-1}\right) \\
\cdots \times T_{k-1}\left(x_{1} \cdots x_{k-1} \mid x_{n+1}\right) \\
\times T_{k}\left(x_{1}, \cdots, x_{k-1}, x_{n+1} \mid x_{k+1}\right) \\
\times T_{k+1}\left(x_{1}, \cdots, x_{k-1}, x_{n+1}, x_{k+1} \mid x_{k+2}\right) \\
\cdots \times T_{n-1}\left(x_{1}, \cdots, x_{k-1}, x_{n+1}, x_{k+1} \cdots x_{n-1} \mid x_{n}\right) \\
T_{n}\left(x_{1}, \cdots, x_{k-1}, x_{n+1}, x_{k+1} \cdots x_{n-1} \mid x_{k}\right),
\end{array}
$$

where now $k=2, \cdots n$. Performing the integrals $\int d x_{k} d x_{k+1} \cdots d x_{n}$ to both objects, using the normalization condition $\int d x_{j} T_{i}\left(x_{1}, \cdots x_{i} \mid x_{j}\right)=1$, and simplifying the factor $P_{k-1}\left(x_{1} \cdots x_{k-1}\right)$, from Eq. (A1) it follows the condition

$$
\begin{array}{r}
T_{k-1}\left(x_{1} \cdots x_{k-1} \mid x_{n+1}\right)=\int d x_{k} \cdots d x_{n} \\
T_{k-1}\left(x_{1} \cdots x_{k-1} \mid x_{k}\right) \\
\times T_{k}\left(x_{1}, \cdots x_{k} \mid x_{k+1}\right) \\
\times T_{k+1}\left(x_{1}, \cdots, x_{k}, x_{k+1} \mid x_{k+2}\right) \\
\cdots \times T_{n-1}\left(x_{1}, \cdots x_{n-1} \mid x_{n}\right) \\
\times T_{n}\left(x_{1}, \cdots x_{n} \mid x_{n+1}\right) .
\end{array}
$$

For $k=n$, this equation reduces to

$$
\begin{aligned}
T_{n-1}\left(x_{1} \cdots x_{n-1} \mid x_{n+1}\right)= & \int d x_{n} T_{n-1}\left(x_{1} \cdots x_{n-1} \mid x_{n}\right) \\
& \times T_{n}\left(x_{1}, \cdots x_{n} \mid x_{n+1}\right) . \quad(\text { A3 }
\end{aligned}
$$


For $k=n-1$, after using the validity of Eq. (A3), Eq. (A2) leads to

$$
\begin{aligned}
T_{n-2}\left(x_{1} \cdots x_{n-2} \mid x_{n+1}\right)= & \int d x_{n-1} \\
& T_{n-2}\left(x_{1} \cdots x_{n-2} \mid x_{n-1}\right) \\
& \times T_{n-1}\left(x_{1}, \cdots x_{n-1} \mid x_{n+1}\right) .
\end{aligned}
$$

Notice that this equation has the same structure as Eq. (A3). Hence, it is simple to realize that Eq. (A2) is satisfied if

$$
\begin{aligned}
T_{n-j}\left(x_{1} \cdots x_{n-j} \mid x_{n+1}\right)= & \int d x_{n-j+1} \\
& T_{n-j}\left(x_{1} \cdots x_{n-j} \mid x_{n-j+1}\right) \\
& \times T_{n-j+1}\left(x_{1}, \cdots x_{n-j+1} \mid x_{n+1}\right) .
\end{aligned}
$$

where $j=1, \cdots n-(k-1)$. This last equation, after a straightforward change of indexes, recovers Eq. (77).

\section{Appendix B: Additive memory case}

The symmetry of the transition probability $T_{n}\left(x_{1} \cdots x_{n} \mid x_{n+1}\right)$ on the previous conditional values $x_{1} \cdots x_{n}$ is trivially fulfilled by assuming that it depends on the addition of these values. Then, we write

$$
T_{n}\left(x_{1} \cdots x_{n} \mid x_{n+1}\right)=\mathcal{T}_{n}\left(x_{1}+x_{2} \cdots+x_{n} \mid x_{n+1}\right),
$$

where $\mathcal{T}_{n}\left(x^{\prime} \mid x\right)$ is a set of equivalent functions that only depends on two arguments. For random variables $\left\{X_{i}\right\}_{i=1}^{n}$ with a finite support, $X \in\left[x_{<}, x_{>}\right]$, the variable $x^{\prime}$ in $\mathcal{T}_{n}\left(x^{\prime} \mid x\right)$ runs in the interval $\left[n x_{<}, n x_{>}\right]$.

From Eq. (77), it follows that the functions $\mathcal{T}_{n}\left(x^{\prime} \mid x\right)$ must to satisfy the recursive relations

$$
\mathcal{T}_{n-1}\left(x^{\prime} \mid x\right)=\int d y \mathcal{T}_{n-1}\left(x^{\prime} \mid y\right) \mathcal{T}_{n}\left(x^{\prime}+y \mid x\right) .
$$

Below we show that the additive assumption allows us to find a complete solution of the hierarchy (7) after assuming different statistics for each single variable.

\section{Gaussian random variables}

For the single distribution of each random variable, let assume a Gaussian distribution

$$
P_{1}(x)=\frac{1}{\sqrt{2 \pi \sigma^{2}}} \exp \left[-\frac{x^{2}}{2 \sigma^{2}}\right]
$$

which satisfies $\int d x P_{1}(x)=1$. The width $\sigma^{2}$ is a free parameter. Given that $\mathcal{T}_{1}\left(x^{\prime} \mid x\right)=T_{1}\left(x^{\prime} \mid x\right)$, in order to fulfill Eq. (5) we assume that $\mathcal{T}_{1}\left(x^{\prime} \mid x\right)$ is a Gaussian distribution in both variables $x^{\prime}$ and $x$. Hence, $\mathcal{T}_{1}\left(x^{\prime} \mid x\right) \approx \exp \left[-\left(\frac{x^{2}}{2 \rho^{2}}+\frac{x^{\prime 2}}{2 \mu^{2}}+\frac{x x^{\prime}}{\nu}\right)\right]$. The undetermined free parameters $(\rho, \mu, \nu)$ are constrained by the normalization condition $\int d x \mathcal{T}_{1}\left(x^{\prime} \mid x\right)=1$, and Eq. (15). After imposing these constraints, we obtain

$$
\mathcal{T}_{1}\left(x^{\prime} \mid x\right)=\frac{1}{\sqrt{2 \pi \rho^{2}}} \exp \left[-\frac{1}{2 \rho^{2}}\left(x-\lambda x^{\prime}\right)^{2}\right],
$$

where the real parameter $\lambda$ is

$$
\lambda \equiv \sqrt{1-\frac{\rho^{2}}{\sigma^{2}}}
$$

$\rho$ remains as a free parameter and satisfies $\rho^{2} \leq \sigma^{2}$. Notice that when $\lambda=0$, that is $\rho=\sigma$, we get independent variables, $\mathcal{T}_{1}\left(x^{\prime} \mid x\right)=P_{1}(x)$. On the other hand, for $\lambda=1$, $\rho \rightarrow 0$, it follows $\mathcal{T}_{1}\left(x^{\prime} \mid x\right)=\delta\left(x-x^{\prime}\right)$. This is the maximal correlated case, where $x=x^{\prime}$. Hence, after the first random value, the next one is equal to the previous one.

Higher transition probabilities can be obtained from Eq. (B2) and the solution (B4). Proposing a Gaussian structure for higher objects, we get

$$
\mathcal{T}_{n}\left(x^{\prime} \mid x\right)=\frac{1}{\sqrt{2 \pi \rho_{n}^{2}}} \exp \left[-\frac{1}{2 \rho_{n}^{2}}\left(x-\lambda_{n} x^{\prime}\right)^{2}\right],
$$

where the coefficients satisfy the recursive relations

$$
\lambda_{n}=\frac{\lambda_{n-1}}{1+\lambda_{n-1}}, \quad \rho_{n}^{2}=\left[1-\left(\frac{\lambda_{n-1}}{1+\lambda_{n-1}}\right)^{2}\right] \rho_{n-1}^{2},
$$

$(n \geq 2)$, with $\lambda_{1} \equiv \lambda$ and $\rho_{1} \equiv \rho$. Their solution is

$$
\lambda_{n}=\frac{\lambda}{1+(n-1) \lambda}, \quad \rho_{n}^{2}=\left[1+\frac{(n-1) \lambda^{2}}{1+n \lambda}\right]^{-1} \rho^{2} .
$$

The joint probability distribution $P_{n}\left(x_{1}, \cdots x_{n}\right)$ can be obtained from the set of transition probabilities [Eq. (4)]. For example, the joint probability $P_{2}\left(x_{1}, x_{2}\right)$, from Eqs. (B3) and (B4), reads

$$
P_{2}\left(x_{1}, x_{2}\right)=\frac{1}{2 \pi \sqrt{\sigma^{2} \rho^{2}}} \exp \left[-\frac{1}{2 \rho^{2}}\left(x_{1}^{2}+x_{2}^{2}-2 \lambda x_{1} x_{2}\right)\right],
$$

which consistently is symmetric in $x_{1}$ and $x_{2}$. For arbitrary $n \geq 2$, we get

$$
P_{n}\left(x_{1}, \cdots x_{n}\right)=\sqrt{\frac{\operatorname{det}\left[A^{(n)}\right]}{(2 \pi)^{n}}} \exp \left[-\frac{1}{2} \sum_{i, j=1}^{n} x_{i} \mathrm{~A}_{i j}^{(n)} x_{j}\right]
$$

where the matrix elements are

$$
\mathrm{A}_{i i}^{(n)}=\frac{1}{\rho_{n-1}^{2}}, \quad \mathrm{~A}_{i j}^{(n)}=-\frac{\lambda_{n-1}}{\rho_{n-1}^{2}}, \quad i \neq j,
$$

where $\rho_{n}$ and $\lambda_{n}$ are defined by Eq. (B8). The determinant of the matrix $\mathrm{A}_{i j}^{(n)}$ reads

$$
\operatorname{det}\left[\mathrm{A}^{(n)}\right]=\left\{[1+(n-1) \lambda] \sigma^{2}\left(\frac{\rho^{2}}{1+\lambda}\right)^{n-1}\right\}^{-1}
$$


The validity of Eq. (B10) can be probe by using the mathematical principle of induction and the recursive relations (B7).

We remark that Eq. (B10) was derived over the basis of the conditional probabilities densities ( $\underline{\mathrm{B} 6}$ ), which in turn are a solution of the hierarchy (B2) after assuming the Gaussian statistics defined by Eq. (B3). Clearly, due to the symmetry of the covariance matrix (B11), the multidimensional Gaussian density (B10) is compatible with the interchangeability symmetry.

Now we obtain the distribution of $W$ [Eq. (26) $]$ for a set of random variables $\left\{X_{i}\right\}_{i=1}^{n}$ correlated in agreement with the Gaussian distribution Eq. (B10), which in turn is related to the transition probability Eq. (B6). The (multiple) Fourier transform of Eq. (B10) reads

$$
G_{k}\left(k_{1}, \cdots k_{k}\right)=\exp \left[-\frac{1}{2} \sum_{i, j=1}^{k} k_{i}\left(1 / \mathrm{A}^{(k)}\right)_{i j} k_{j}\right],
$$

where $\left(1 / \mathrm{A}^{(k)}\right)$ is the matrix inverse of $\mathrm{A}^{(k)}$ [Eq. (B11)]. It can be written as

$$
\left(1 / \mathrm{A}^{(k)}\right)_{i i}=\sigma^{2}, \quad\left(1 / \mathrm{A}^{(k)}\right)_{i j}=\sigma^{2} \lambda, \quad i \neq j,
$$

where $\lambda=\left(1-\rho^{2} / \sigma^{2}\right)^{1 / 2}[$ Eq. (B5) $]$. Hence, from Eqs. (28) and (B13), we get

$$
G_{w_{n}}(k)=\exp \left\{-\frac{1}{2} \sigma^{2} \lambda k^{2}\left[1+\frac{1}{n}\left(\lambda^{-1}-1\right)\right]\right\} .
$$

After taking the limit $n \rightarrow \infty$, it follows

$$
P(w)=\sqrt{\frac{1}{2 \pi \sigma^{2} \lambda}} \exp \left[-\frac{1}{2} \frac{w^{2}}{\sigma^{2} \lambda}\right] .
$$

Contrarily to the case of independent variables, here the distribution of $W$ is not a delta Dirac distribution, Eq. (34). This departure has its origin in the correlations between the random variables, which are tuned by the parameter $\lambda$. In fact, in the limit $\lambda \rightarrow 0$ we recover Eq. (34) with $\bar{x}=0$, that is, independent variables. On the other hand, for maximally correlated variables, $\lambda \rightarrow 1$, we recover the Gaussian distribution $P_{1}(x)$ [Eq. (B3)]. This result, which gives the maximal departure with respect to independent variables, follows after noting that all random variables assume the same value [see the transition probabilities Eqs. (B4) and (B6)].

\section{Linear additive memory case}

Here, we search another class of solution which in addition assume that the transition probabilities $\mathcal{T}_{n}\left(x^{\prime} \mid x\right)$ depend linearly on the argument $x^{\prime}$. In the following results, the structure of $P_{1}(x)$ is arbitrary.

Given $P_{1}(x)$, and given the linear dependence of $\mathcal{T}_{1}\left(x^{\prime} \mid x\right)$ on $x^{\prime}$, the relation defined by Eq. (5) becomes

$$
\mathcal{T}_{1}(\langle X\rangle \mid x)=P_{1}(x), \quad\langle X\rangle \equiv \int d x P_{1}(x) x .
$$

Given $P_{1}(x)$, any transition probability density $T_{1}\left(x^{\prime} \mid x\right)$ satisfying this equation is a valid one. On the other hand, assuming that all transition probability densities depend linearly on $x^{\prime}$, the conditions (B2) can be written as

$$
\mathcal{T}_{n}\left(x^{\prime}+\langle X\rangle_{n-1, x^{\prime}} \mid x\right)=\mathcal{T}_{n-1}\left(x^{\prime} \mid x\right),
$$

where the conditional average $\langle X\rangle_{n-1, x^{\prime}}$ is defined as

$$
\langle X\rangle_{n-1, x^{\prime}} \equiv \int d x \mathcal{T}_{n-1}\left(x^{\prime} \mid x\right) x .
$$

By evaluating the previous two expressions in $x^{\prime}=\langle X\rangle$, it follows the relation

$$
\mathcal{T}_{n}(n\langle X\rangle \mid x)=P_{1}(x),
$$

which generalize that defined by Eq. (B17).

From Eq. (B19), we realize that $\langle X\rangle_{n-1, x^{\prime}}$ is also a linear function of $x^{\prime}$. In particular, it is possible to write

$$
\langle X\rangle_{1, x^{\prime}}=\int d x \mathcal{T}_{1}\left(x^{\prime} \mid x\right) x=a x^{\prime}+b .
$$

This equation defines the constants $a$ and $b$, the former being a dimensionless one, while the last one has units of $x$. Multiplying the previous expression by $P_{1}\left(x^{\prime}\right)$ and integrating in $x^{\prime}$ it follows the relation $\langle X\rangle=b /(1-a)$.

From (B21), the solution of Eq. (B18) for $n=2$ is $\mathcal{T}_{2}\left(x^{\prime}(1+a)+b \mid x\right)=\mathcal{T}_{1}\left(x^{\prime} \mid x\right)$, which can be rewritten as

$$
\mathcal{T}_{2}\left(x^{\prime} \mid x\right)=\mathcal{T}_{1}\left(\frac{x^{\prime}-b}{1+a} \mid x\right) .
$$

In a similar form, an explicit expression for $\mathcal{T}_{3}\left(x^{\prime} \mid x\right)$ can be obtained. For arbitrary $n$, as a solution of Eq. (B18) we propose the expression

$$
\mathcal{T}_{n}\left(x^{\prime} \mid x\right)=\mathcal{T}_{1}\left(\frac{x^{\prime}-(n-1) b}{1+(n-1) a} \mid x\right) .
$$

The validity of this result can be prove from Eq. (B18) by using the mathematical principle of induction.

\section{Discrete distributions with finite support}

The set of functions defined by Eq. (B23) give a full solution to the hierarchical structure (B2). Nevertheless, it is not guaranteed that their are positive functions. In order to check this issue, we consider discrete random variables defined by

$$
P_{1}(x)=\sum_{\mu=1}^{M} q_{\mu} \delta\left(x-x_{\mu}\right)
$$

where the positive weights satisfy $\sum_{\mu=1}^{M} q_{\mu}=1$.

The mean value, $\langle X\rangle=\int d x P_{1}(x) x$, reads $\langle X\rangle=$ $\sum_{\mu=1}^{M} q_{\mu} x_{\mu}$. The first conditional density, given it linear dependence on $x^{\prime}$, is written as

$$
\mathcal{T}_{1}\left(x^{\prime} \mid x\right)=\frac{1}{\mathcal{N}} \sum_{\mu=1}^{M}\left(\alpha_{\mu}+\beta_{\mu} x^{\prime}\right) \delta\left(x-x_{\mu}\right),
$$


where $\left(\alpha_{\mu}, \beta_{\mu}\right)$ and $\mathcal{N}$ are arbitrary parameters. Using the normalization condition $\int d x \mathcal{T}_{1}\left(x^{\prime} \mid x\right)=1$, it follows $\mathcal{N}=\sum_{\mu=1}^{M} \alpha_{\mu}$, and

$$
\sum_{\mu=1}^{M} \beta_{\mu}=0
$$

The condition $\mathcal{T}_{1}(\langle X\rangle \mid x)=P_{1}(x)$, leads to $\frac{1}{\mathcal{N}}\left(\alpha_{\mu}+\right.$ $\left.\beta_{\mu}\langle X\rangle\right)=q_{\mu}$. Under the association $\left(\beta_{\mu} / \mathcal{N}\right) \rightarrow \beta_{\mu}$, we get

$$
\mathcal{T}_{1}\left(x^{\prime} \mid x\right)=\sum_{\mu=1}^{M}\left[q_{\mu}+\beta_{\mu}\left(x^{\prime}-\langle X\rangle\right)\right] \delta\left(x-x_{\mu}\right) .
$$

The first conditional average reads

$$
\int d x \mathcal{T}_{1}\left(x^{\prime} \mid x\right) x=\zeta x^{\prime}+\langle X\rangle(1-\zeta)=a x^{\prime}+b,
$$

where the constant $\zeta$ is

$$
\zeta \equiv \sum_{\mu=1}^{M} x_{\mu} \beta_{\mu} .
$$

From Eq. (B23), higher objects reads

$$
\mathcal{T}_{n}\left(x^{\prime} \mid x\right)=\sum_{\mu=1}^{M}\left[q_{\mu}+\beta_{\mu} \frac{x^{\prime}-n\langle X\rangle}{1+(n-1) \zeta}\right] \delta\left(x-x_{\mu}\right) .
$$

We remark that this set of equations provide a solution to the full hierarchy of conditional probabilities under the interchangeability symmetry. Nevertheless, the positivity of these objects must to be checked.

The constants $\beta_{\mu}$ should be chosen such that the positivity of $\mathcal{T}_{n}\left(x^{\prime} \mid x\right)$ is guaranteed for all $n$ and $x^{\prime} \in$ $\left(n x_{<}, n x_{>}\right)$, where $x_{<}$and $x_{>}$define respectively the minimal and maximal values of the set $\left\{x_{\mu}\right\}_{\mu=1}^{M}$. Hence, for $n=1$ it follows

$$
q_{\mu}+\beta_{\mu}(x-\langle X\rangle) \geq 0,
$$

while in the limit $n \rightarrow \infty$, we get

$$
q_{\mu}+\frac{\beta_{\mu}(x-\langle X\rangle)}{\sum_{\nu=1}^{M} x_{\nu} \beta_{\nu}} \geq 0 .
$$

In both inequalities, $x$ assume values over the set $\left\{x_{u}\right\}$. In the case of two states, $M=2$, from these inequalities we obtain $\beta \leq\left|x_{2}-x_{1}\right|^{-1}$, where $\beta_{1}=-\beta_{2}=\beta$, and $\left\{x_{\mu}\right\}=$ $\left\{x_{1}, x_{2}\right\}$. Hence, positivity can always be guaranteed in this case.

In general for $M \geq 3$, there is not a solution for the set $\left\{\beta_{\mu}\right\}$ that guarantees the validity of the previous two inequalities. In fact, from Eq. (B32), we deduce that

$$
\left|\beta_{\mu}(x-\langle X\rangle)\right| \leq q_{\mu}\left|\sum_{\nu=1}^{M} x_{\nu} \beta_{\nu}\right| .
$$

Taking $x \rightarrow x_{\mu}$, and adding in the $\mu$ index, $\sum_{\mu=1}^{M}$, it follows

$$
\sum_{\nu=1}^{M}\left|\beta_{\mu} x_{\mu}\right| \leq\left|\sum_{\nu=1}^{M} x_{\mu} \beta_{\mu}\right|
$$

Hence, we deduce that $x_{\mu} \beta_{\mu}>0$, and then $\sum_{\mu=1}^{M} x_{\mu} \beta_{\mu}>$ 0 . Therefore, Eqs. (B31) and (B32) are equivalents, in the sense that one of them always implies the other. Taking one of them and the previous one, it follows $M(M-1)-1$ equations, while the number of variables is $M-1$. Thus, a consistent solution (positive transition probabilities) is only available when $M=2$.

For classical spin variables $x_{\mu}= \pm 1$, parametrizing $\beta=(1 / 2)(1+\lambda)^{-1} \leq\left|x_{+}-x_{-}\right|^{-1}=1 / 2$, from Eq. (B30) we get $\left(\lambda_{ \pm}=\lambda q_{ \pm}\right)$

$$
\mathcal{T}_{n}\left(x^{\prime} \mid x\right)=\left(\frac{\lambda_{+}+\frac{n+x^{\prime}}{2}}{n+\lambda}\right) \delta(x-1)+\left(\frac{\lambda_{-}+\frac{n-x^{\prime}}{2}}{n+\lambda}\right) \delta(x+1) .
$$

This expression gives a positive solution consistent with interchangeability. Nevertheless, it is simple to realize that the quantities $\frac{n+x^{\prime}}{2}$ and $\frac{n-x^{\prime}}{2}$ give the number of times $n_{+}$and $n_{-}$that the previous variables assumed the values \pm 1 respectively. Therefore, Eq. (B35) recovers the transition probability corresponding to the Pólya urn scheme, Eq. (12).

\section{Appendix C: de Finetti representation}

de Finetti [21] introduced the concept of interchangeability and also defined a general representation structure for the joint probability density of a set of dichotomic interchangeable variables. The de Finetti representation can be generalized for arbitrary (non-dichotomic) random variables. Given a set of interchangeable random variables $\left\{X_{i}\right\}_{i=1}^{n}$, their $n$-joint probability density is expressed as

$$
P_{n}\left(x_{1}, \cdots, x_{n}\right)=\int_{\Omega_{y}} d y p(y) \prod_{i=1}^{n} p\left(y \mid x_{i}\right) .
$$

Here, $p(y)$ is the probability density of an extra random variable $Y$, which assume values in the domain $\Omega_{y}$. On the other hand, $p\left(y \mid x_{i}\right)$ is a transition probability: it gives the probability density of $X_{i}$ given the value $y$ of the random variable $Y$.

The structure given by Eq. (C1) allows us to read the realizations of the correlated set $\left\{X_{i}\right\}_{i=1}^{n}$ as an average over realizations of a set of identical random variables with the joint probability density $\prod_{i=1}^{n} p\left(y \mid x_{i}\right)$. A similar interpretation can be obtained for the transition density $T_{n}\left(x_{1}, \cdots x_{n} \mid x_{n+1}\right)$. In fact, by using that $T_{n}\left(x_{1}, \cdots x_{n} \mid x_{n+1}\right)=$ $P_{n+1}\left(x_{1}, \cdots, x_{n+1}\right) / P_{n}\left(x_{1}, \cdots, x_{n}\right)$, it can be written as

$$
T_{n}\left(x_{1}, \cdots x_{n} \mid x_{n+1}\right)=\int_{\Omega_{y}} d y p_{n}\left(\left\{x_{i}\right\} \mid y\right) p\left(y \mid x_{n+1}\right),
$$

where $p\left(y \mid x_{n+1}\right)$ was introduced previously while $p_{n}\left(\left\{x_{i}\right\} \mid y\right)$ is

$$
p_{n}\left(\left\{x_{i}\right\} \mid y\right)=\frac{\prod_{i=1}^{n} p\left(y \mid x_{i}\right)}{\int_{\Omega_{y}} d y^{\prime} p\left(y^{\prime}\right) \prod_{j=1}^{n} p\left(y^{\prime} \mid x_{j}\right)} p(y) .
$$


Therefore, $T_{n}\left(x_{1}, \cdots x_{n} \mid x_{n+1}\right)$ is set by $p\left(y \mid x_{n+1}\right)$, where now the statistical distribution $p_{n}\left(\left\{x_{i}\right\} \mid y\right)$ of the random variable $Y$ [see Eq. (C2)] depends on all previous values $\left\{X_{i}\right\}_{i=1}^{n}$. Hence, $p_{n}\left(x^{\prime} \mid y\right)$ can be read as the conditional probability density of the random variable $Y$ "given" the previous history defined by the set of values $\left\{x_{i}\right\}_{i=1}^{n}$. On the other hand, it is simple to check that Eq. (C2) satisfies the hierarchical equations defined by Eq. (7).

The sum variable (26) can be straightforwardly characterized from Eqs. (C1) and (29). We get

$$
G_{w_{n}}(k)=\int_{\Omega_{y}} \operatorname{dyp}(y)[G(y \mid k / n)]^{n},
$$

where $G(y \mid k) \equiv \int_{-\infty}^{+\infty} d x e^{i k x} p(y \mid x)$. In the asymptotic limit, assuming valid the law of large numbers for the transition $p(y \mid x)$, from Eqs. (30) and (34) it follows

$$
P(w)=\int_{\Omega_{y}} d y p(y) \delta\left(w-\bar{x}_{y}\right),
$$

where the mean value $\bar{x}_{y}$ is a function of $y$,

$$
\bar{x}_{y} \equiv \int d x p(y \mid x) x .
$$

In the case of dichotomic variables, $X_{i}=0,1$, with transition probability $p\left(y \mid x_{i}\right)=y^{x_{i}}(1-y)^{1-x_{i}}$, the joint probability $P_{n}\left(x_{1}, \cdots, x_{n}\right)$, from Eq. (C1), becomes

$$
P_{n}\left(x_{1}, \cdots, x_{n}\right)=\int_{0}^{1} d y p(y) \prod_{i=1}^{n} y^{x_{i}}(1-y)^{1-x_{i}} .
$$

Noting that the dependence of the probability $P_{n}\left(x_{1}, \cdots, x_{n}\right)$ on the set $\left\{x_{i}\right\}_{i=1}^{n}$ can be written in terms of the the variable $x^{\prime} \equiv \sum_{i=1}^{n} x_{i}$ [Eq. [C7)], from Eq. (C2) it follows the presentation

$$
\mathcal{T}_{n}\left(x^{\prime} \mid x\right)=\int_{0}^{1} d y p_{n}\left(x^{\prime} \mid y\right) y^{x}(1-y)^{1-x},
$$

where

$$
p_{n}\left(x^{\prime} \mid y\right)=\frac{y^{x^{\prime}}(1-y)^{n-x^{\prime}}}{\int_{0}^{1} d \tilde{y} p(\tilde{y}) \tilde{y}^{x^{\prime}}(1-\tilde{y})^{n-x^{\prime}}} p(y) .
$$

Eq. (C8) provides a representation for the transition probability $\mathcal{T}_{n}\left(x^{\prime} \mid x\right)$ similar to that defined by Eq. (C7).

Given that Eq. (C6) leads to $\bar{x}_{y}=y$, from Eq. (C5) it follows that $P(w)=\left.p(y)\right|_{y=w}$. Hence, any attractor can be obtained by choosing an arbitrary density $p(y)$.

If one choose a Beta distribution

$$
p(y)=\frac{\Gamma\left(\alpha+\alpha^{\prime}\right)}{\Gamma(\alpha) \Gamma\left(\alpha^{\prime}\right)} y^{\alpha-1}(1-y)^{\alpha^{\prime}-1},
$$

where $\alpha>1$ and $\alpha^{\prime}>1$ are real parameters, from Eqs. (C7) it is possible to obtain the joint probability densities. In particular, $P_{1}(x)$ can be written as $P_{1}(x)=$ $\left[\alpha^{\prime} \delta(x)+\alpha \delta(x-1)\right] /\left(\alpha+\alpha^{\prime}\right)$. On the other hand, by rewriting Eq. (C8) as $\mathcal{T}_{n}\left(x^{\prime} \mid x\right)=\delta(x) \int_{0}^{1} d y p_{n}\left(x^{\prime} \mid y\right)(1-$ $y)+\delta(x-1) \int_{0}^{1} d y p_{n}\left(x^{\prime} \mid y\right) y$, the transition probability density explicitly reads

$$
\mathcal{T}_{n}\left(x^{\prime} \mid x\right)=\frac{\left(n-x^{\prime}+\alpha^{\prime}\right) \delta(x)+\left(x^{\prime}+\alpha\right) \delta(x-1)}{n+\alpha+\alpha^{\prime}} .
$$

In deriving this expression we used the dichotomic property of the random variables.

By introducing the parameter $\lambda=\alpha+\alpha^{\prime}$, the weights $q_{0}=\alpha^{\prime} /\left(\alpha+\alpha^{\prime}\right), q_{1}=\alpha /\left(\alpha+\alpha^{\prime}\right)$, and the numbers $n_{0}=n-x^{\prime}, n_{1}=x^{\prime}$, the transition probability (C11) can be written as a particular case of the Pólya urn scheme [see Eq. [12)]. In fact, $n_{0}$ and $n_{1}$ are the number of times that the random variables assumed the values 0 and 1 respectively.

The probability of the variable $X^{\prime} \equiv \sum_{i=1}^{n} X_{i}$, from Eqs. (C7) and (C10) reads

$$
P\left(x^{\prime}\right)=\left(\begin{array}{c}
n \\
x^{\prime}
\end{array}\right) \frac{\Gamma\left(\alpha+\alpha^{\prime}\right)}{\Gamma\left(n+\alpha+\alpha^{\prime}\right)} \frac{\Gamma\left(n-x^{\prime}+\alpha^{\prime}\right)}{\Gamma\left(\alpha^{\prime}\right)} \frac{\Gamma\left(x^{\prime}+\alpha\right)}{\Gamma(\alpha)},
$$

where $\Gamma(x)$ is the Gamma function. The factor $\left(\begin{array}{c}n \\ x^{\prime}\end{array}\right)$ follows from all configurations that lead to the same value of $x^{\prime}$.

\section{Appendix D: Blackwell-MacQueen urn scheme}

Here, we obtain the joint probability of the BlackwellMacQueen urn scheme [Eq. (10)], as well as the characteristic function of the sum variable.

The probability density of $X_{1}$ is $P_{1}\left(x_{1}\right)$. The second joint probability density, from Eq. (4), reads

$$
P_{2}\left(x_{1}, x_{2}\right)=\frac{\lambda p\left(x_{1}\right) p\left(x_{2}\right)+p\left(x_{1}\right) \delta\left(x_{2}-x_{1}\right)}{(1+\lambda)} .
$$

Furthermore,

$$
\begin{aligned}
P_{3}\left(x_{1}, x_{2}, x_{3}\right)= & \frac{1}{(1+\lambda)(2+\lambda)}\left[\lambda^{2} p\left(x_{1}\right) p\left(x_{2}\right) p\left(x_{3}\right)\right. \\
& +\lambda p\left(x_{1}\right) p\left(x_{2}\right) \delta\left(x_{3}-x_{2}\right) \\
& +\lambda p\left(x_{1}\right) \delta\left(x_{2}-x_{1}\right) p\left(x_{3}\right) \\
& +\lambda p\left(x_{1}\right) p\left(x_{2}\right) \delta\left(x_{3}-x_{1}\right) \\
& \left.+2 p\left(x_{1}\right) \delta\left(x_{2}-x_{1}\right) \delta\left(x_{3}-x_{1}\right)\right] .
\end{aligned}
$$

In general, we can write

$$
P_{n}\left(x_{1}, \cdots x_{n}\right)=\sum_{\pi \in \Pi_{n}} w_{n}(\pi) \mathcal{P}_{\pi}^{n}\left(x_{1}, \cdots x_{n}\right),
$$

where $\pi$ runs through the set $\Pi_{n}$ of all partitions of $n$ positive integers. Each partition $\pi$ is characterized by $n$ positive natural numbers $\left\{m_{1}, m_{2}, \cdots, m_{n}\right\}$, which satisfy a Diofantine equation

$$
1 \cdot m_{1}+2 \cdot m_{2}+3 \cdot m_{3}+\cdots n \cdot m_{n}=n
$$


which in fact is a Frobenious equation. The symmetry condition is consistent with $w_{n}(\pi)=w_{n}\left(\left\{m_{i}\right\}\right)$. Therefore, we can write

$$
P_{n}\left(x_{1}, \cdots x_{n}\right)=\sum_{\left\{m_{i}\right\}} w_{n}\left(\left\{m_{i}\right\}\right) \mathcal{P}_{\left\{m_{i}\right\}}^{(n)}\left(x_{1}, \cdots x_{n}\right) .
$$

By using the mathematical principle of induction, it is possible to obtain

$$
w_{n}\left(\left\{m_{i}\right\}\right)=\frac{(n+\lambda)}{\lambda} \prod_{i=1}^{n} \frac{[(i-1) ! \lambda]^{m_{i}}}{(i+\lambda)},
$$

which can be rewritten as

$$
w_{n}\left(\left\{m_{i}\right\}\right)=\frac{\Gamma(\lambda)}{\Gamma(n+\lambda)} \prod_{i=1}^{n}[\lambda(i-1) !]^{m_{i}} .
$$

On the other hand, for each set of numbers $\left\{m_{i}\right\}$ the corresponding probability reads

$$
\begin{aligned}
\mathcal{P}_{\{m i\}}^{(n)}\left(x_{1}, \cdots x_{n}\right) & =\sum_{\{\chi\}}\left\{\prod_{i_{1}=1}^{m_{1}} P_{1}\left(\chi_{i_{1}}\right)\right. \\
& \times \prod_{i_{2}=1}^{m_{2}} P_{1}\left(\chi_{i_{2}}\right) \delta\left(\chi_{i_{2}}-\chi_{i_{2}}^{(1)}\right) \\
\times & \prod_{i_{3}=1}^{m_{3}} P_{1}\left(\chi_{i_{3}}\right) \delta\left(\chi_{i_{3}}-\chi_{i_{3}}^{(1)}\right) \delta\left(\chi_{i_{3}}-\chi_{i_{3}}^{(2)}\right) \\
& \left.\cdots \times \prod_{i_{n}=1}^{m_{n}} P_{1}\left(\chi_{i_{n}}\right) \prod_{j=1}^{n-1} \delta\left(\chi_{i_{n}}-\chi_{i_{n}}^{(j)}\right)\right\} .
\end{aligned}
$$

The set of variables $\left\{\chi_{i}\right\}$ assume values over the set $\left\{x_{i}\right\}_{i=1}^{n}$. In each product there are $m_{j}$ independent variables $\chi_{i_{j}}$, each one having associated other different $j$ variables $\chi_{i_{j}}^{(j)}$ that, due to the delta-Dirac contributions, assume the same value than $\chi_{i_{j}}$. Hence, in the previous expression we have in total $m_{1}+2 m_{2}+3 m_{3}+\cdots n m_{n}=n$ different variables $\chi$. The sum runs overs all possible set of variables $\chi \rightarrow x$ that lead to a different contribution. Consequently, the sum $\sum_{\{\chi\}}$ has a number $\mathcal{N}\left(\left\{m_{i}\right\}\right)$ of different terms, where

$$
\mathcal{N}\left(\left\{m_{i}\right\}\right)=\frac{n !}{m_{1} !(1 !)^{m_{1}} m_{2} !(2 !)^{m_{2}} \cdots m_{n} !(n !)^{m_{n}}} .
$$

This number follows by taking into account that $\mathcal{P}_{\{m i\}}^{(n)}\left(x_{1}, \cdots x_{n}\right)$ does not depends on the order that the variables appear.

From the previous results, we can study the statistics of the sum variable Eq. (26). For simplifying the notation $G_{1}(k) \rightarrow G(k)$. From the multiple Fourier transform Eqs. (28) and (29), it follows

$$
G_{w}^{(2)}(2 k)=\frac{\lambda G^{2}(k)+G(2 k)}{(1+\lambda)} .
$$

Similarly,

$$
G_{w}^{(3)}(3 k)=\frac{\lambda^{2} G^{3}(k)+3 \times \lambda^{2} G(k) G(2 k)+2 G(3 k)}{(1+\lambda)(2+\lambda)} .
$$

In general, from Eq. (D4) we obtain

$$
G_{w}^{(n)}(n k)=\sum_{\left\{m_{i}\right\}} w_{n}\left(\left\{m_{i}\right\}\right) \mathcal{N}\left(\left\{m_{i}\right\}\right) \prod_{i=1}^{n}[G(i k)]^{m_{i}}
$$

This expression explicitly reads

$$
G_{w}^{(n)}(n k)=\frac{n ! \Gamma(\lambda)}{\Gamma(n+\lambda)} \sum_{\left\{m_{i}\right\}} \prod_{i=1}^{n} \frac{1}{m_{i} !}\left[\lambda \frac{G(i k)}{i}\right]^{m_{i}}
$$

Now, we note that this expression can be write in terms of a complete Bell polynomial $B_{n}\left(x_{1}, \cdots x_{n}\right)$, which is defined as 28]

$$
B_{n}\left(\left\{x_{i}\right\}\right)=n ! \sum_{\left\{m_{i}\right\}} \prod_{i=1}^{n} \frac{1}{m_{i} !}\left(\frac{x_{i}}{i !}\right)^{m_{i}}
$$

Therefore, under the association $x_{i}=(i-1) ! \lambda G(i k)$, we can write

$$
G_{w}^{(n)}(k)=\frac{\Gamma(\lambda)}{\Gamma(n+\lambda)} B_{n}(\{(i-1) ! \lambda G(i k / n)\}) .
$$

The complete Bell polynomial can be written as a determinant of a matrix, $B_{n}\left(\left\{x_{i}\right\}\right)=B_{n}\left(x_{1}, \cdots x_{n}\right)=\operatorname{det} \mathcal{B}_{n}$,

$\mathcal{B}_{n}=\left(\begin{array}{ccccccc}x_{1} & \left(\begin{array}{c}n-1 \\ 1\end{array}\right) x_{2} & \left.\begin{array}{c}n-1 \\ 2\end{array}\right) x_{3} & \left(\begin{array}{c}n-1 \\ 3\end{array}\right) x_{4} & \cdots & \cdots & x_{n} \\ -1 & x_{1} & \left(\begin{array}{c}n-2 \\ 1\end{array}\right) x_{2} & \left(\begin{array}{c}n-2 \\ 2\end{array}\right) x_{3} & \cdots & \cdots & x_{n-1} \\ 0 & -1 & x_{1} & \left(\begin{array}{c}n-3 \\ 1\end{array}\right) x_{2} & \cdots & \cdots & x_{n-2} \\ 0 & 0 & -1 & x_{1} & \cdots & \cdots & x_{n-3} \\ 0 & 0 & 0 & -1 & \cdots & \cdots & x_{n-4} \\ \vdots & \vdots & \vdots & \vdots & \ddots & \ddots & \vdots \\ 0 & 0 & 0 & 0 & \cdots & -1 & x_{1}\end{array}\right)$,

giving an exact and compact expression for $G_{w}^{(n)}(k)$. It can be evaluated for arbitrary characteristic functions $G(k)$. This urn model also lead to a wide family of probability densities that departs from a delta Dirac distribution, Eq. (34). 
[1] B. V. Gnedenko and A. N. Kolmogorov, Limit distributions for sums of independent random variables, (Addison-Wesley, USA, 1968).

[2] W. Feller, An introduction to probability theory and applications, Vol. I \& II, (John Wiley \& Sons, 1967).

[3] N. G. van Kampen, Stochastic Processes in Physics and Chemistry, (Sec. Ed., North-Holland, Amsterdam, 1992).

[4] C. W. Gardiner, Handbook of Stochastic Methods, (Springer-Verlag, Berlin, 2004).

[5] P. Lévy, Théorie de lÁdditions des Variables Aléatoires, (Gauthier Villars, Paris, 1954).

[6] E. J. Gumbel, Statistics of Extremes (Columbia University Press, New York, 1958); J. -P. Bouchaud and M. Mézard, J. Phys. A 30, 7997 (1997).

[7] E. Bertin, Global Fluctuations and Gumbel Statistics, Phys. Rev. Lett. 95, 170601 (2005); E. Bertin and M. Clusel, Generalized extreme value statistics and sum of correlated variables, J. Phys. A 39, 7607 (2006); M. Clusel and E. Bertin, Interplay between sum and extreme value statistics, Int. J. Mod. Phys. B 22, 3311 (2008).

[8] F. Baldowin and A. L. Stella, Central limit theorem for anomalous scaling due to correlations, Phys. Rev. E 75, 020101(R) (2007).

[9] C. Tsallis, Introduction to Nonextensive Statistical Mechanics, (Springer, 2009).

[10] G. Miritello, A. Pluchino, and A. Rapisarda, Central limit behavior in the Kuramoto model at the "edge of chaos," Phys. A 388, 4818 (2009); A. Pluchino, A. Rapisarda, and C. Tsallis, A closer look at the indications of $q$-generalized Central Limit Theorem behavior in quasistationary states of the HMF model, Phys. A 387, 3121 (2008); A. Pluchino and A. Rapisarda, Nonergodicity and central limit behavior for long-range interactions, Euro Phys. Lett. 80, 26002 (2007).

[11] U. Tirnakli, C. Beck, and C. Tsallis, Central limit behavior of deterministic dynamical systems, Phys. Rev. E 75, 040106(R) (2007); U. Tirnakli, C. Tsallis, and C. Beck, Closer look at time averages of the logistic map at the edge of chaos, Phys. Rev. E 79, 056209 (2009); U. Tirnakli, H. J. Jensen, and C. Tsallis, Restricted random walk model as a new testing ground for the applicability of $q$-statistics, Eur. Phys. Lett. 96, 40008 (2011).

[12] J. Ruseckas, Probabilistic model of $N$ correlated binary random variables and non-extensive statistical mechanics, Phys. Lett. A 379, 654 (2015); D. O'Malley, V. V. Vesselinov, and J. H. Cushman, Diffusive mixing and Tsallis entropy, Phys. Rev. E 91, 042143 (2015).

[13] R. Hanel, S. Thurner, and C. Tsallis, Limit distributions of scale-invariant probabilistic models of correlated random variables with $q$-Gaussian as an explicit example, Eur. Phys. J. B 72, 263 (2009).

[14] A. Rodriguez, V. Schwämmle, and C. Tsallis, Strictly and asymptotically scale invariant probabilistic models of $N$ correlated binary random variables having $q$-Gaussians as $N \rightarrow \infty$ limiting distributions, J. Stat. Mech.: Theory Exp. (2008), P09006; S. Umarov, C. Tsallis, and S. Steinberg, On a $q$-Central Limit Theorem Consistent with Nonextensive Statistical Mechanics, Milan J. Math. 76, 307 (2008).
[15] C. Vignat and A. Plastino, Central limit theorem and deformed exponentials, J. Phys. A 40, F969 (2007); C. Vignat and A. Plastino, Scale invariance and related properties of $q$-Gaussian systems, Phys. Lett. A 365, 370 (2007).

[16] A. Rodríguez and C. Tsallis, A dimension scale-invariant probabilistic model based on Leibniz-like pyramids, J. Math. Phys. 53, 023302 (2012).

[17] E. M. F. Curado, J. P. Gazeau, and L. M. C. S. Rodrígues, On a Generalization of the Binomial Distribution and Its Poisson-like Limit, J. Stat. Phys. 146, 264 (2012); H. Bergeron, E. M. F. Curado, J. P. Gazeau, and L. M. C. S. Rodrigues, Symmetric generalized binomial distributions, J. Math. Phys. 54, 123301 (2013); G. A. Casas, F. D. Nobre, and E. M. F. Curado, Nonlinear Ehrenfest's urn model, Phys. Rev. E 91, 042139 (2015).

[18] H. J. Hilhorst, Central limit theorems for correlated variables: some critical remarks, Brazilian Journal of Physics 39, 371 (2009); H. J. Hilhorst and G. Schehr, A note on $q$-Gaussian and non-Gaussians in statistical mechanics, J. Stat. Mech. P06003 (2007).

[19] C. Beck, Dynamical Foundations of Nonextensive Statistical Mechanics, Phys. Rev. Lett. 87, 180601 (2001); Eur. Phys. Lett. 57, 329 (2002); C. Beck and E. G. D. Cohen, Superstatistics, Phys. A 322, 267 (2003); H. Touchette and C. Beck, Asymptotics of superstatistics, Phys. Rev. E 71, 016131 (2005); S. Abe, C. Beck, and E. G. D. Cohen, Superstatistics, thermodynamics, and fluctuations, Phys. Rev. E 76, 031102 (2007).

[20] A. A. Budini, Extended $q$-Gaussian and $q$-exponential distributions from gamma random variables, Phys. Rev. E 91, 052113 (2015).

[21] B. de Finetti, Funzione caratteristica di un fenomeno aleatorio, Memorie della R. Accademia Nazionale dei Lincei 6, 86 (1930); B. de Finetti, La prévision: ses lois logiques, ses sources subjectives, Ann. Inst. H. Poincaré 7, 1 (1937).

[22] E. T. Jaynes, Probability theory: The Logic of Science, (Cambridge University Press, New York, 2003).

[23] E. Hewitt and L. J. Savage, Symmetric measures on Cartesian products, Trans. Amer. Math. Soc. 80, 470 (1955).

[24] D. Heath and W. Sudderth, de Finetti Theorem on Exchangeable Variables, The American Statistician 30, 188 (1976).

[25] J. F. C. Kingman, Uses of exchangeability, Ann. Prob. 6, 183 (1978).

[26] N. L. Johnson and S. Kotz, Urn Models and Their Application, (John Wiley\&Sons, 1977).

[27] D. Blackwell and J. B. MacQueen, Fergurson distributions via Pólya urn schemes, The Annals of Statistics 1, 353 (1973).

[28] J. Pitman, Combinatorial Stochastic Processes, (Springer 2006).

[29] B. Bassetti, M. Zarei, M. C. Lagomarsino, and G. Bianconi, Statistical mechanics of the "Chinese restaurant process": Lack of self-averaging, anomalous finite-size effects, and condensation, Phys. Rev. E 80, 066118 (2009). 